\title{
Finite-Time Reentry Attitude Control Using Time-Varying Sliding Mode and Disturbance Observer
}

\author{
Xuzhong Wu, Shengjing Tang, Jie Guo, and Yao Zhang \\ Key Laboratory of Dynamics and Control of Flight Vehicle, Ministry of Education, School of Aerospace Engineering, \\ Beijing Institute of Technology, Beijing 100081, China \\ Correspondence should be addressed to Shengjing Tang; tsj8678@126.com
}

Received 5 June 2014; Revised 16 September 2014; Accepted 11 October 2014

Academic Editor: Rongni Yang

Copyright (C) 2015 Xuzhong Wu et al. This is an open access article distributed under the Creative Commons Attribution License, which permits unrestricted use, distribution, and reproduction in any medium, provided the original work is properly cited.

\begin{abstract}
This paper presents the finite-time attitude control problem for reentry vehicle with redundant actuators in consideration of planet uncertainties and external disturbances. Firstly, feedback linearization technique is used to cancel the nonlinearities of equations of motion to construct a basic mode for attitude controller. Secondly, two kinds of time-varying sliding mode control methods with disturbance observer are integrated with the basic mode in order to enhance the control performance and system robustness. One method is designed based on boundary layer technique and the other is a novel second-order sliding model control method. The finite-time stability analyses of both resultant closed-loop systems are carried out. Furthermore, after attitude controller produces the torque commands, an optimization control allocation approach is introduced to allocate them into aerodynamic surface deflections and on-off reaction control system thrusts. Finally, the numerical simulation results demonstrate that both of the time-varying sliding mode control methods are robust to uncertainties and disturbances without chattering phenomenon. Moreover, the proposed second-order sliding mode control method possesses better control accuracy.
\end{abstract}

\section{Introduction}

Covering from outer space into earth's atmosphere, reentry flight is a critical phase of the operation for reentry vehicles (RVs) [1]. Since the flight conditions change rapidly in the reentry phase, reentry attitude control is always in face of wide range of planet uncertainties and external disturbances. On the other hand, aerodynamic surfaces come into the eyes of engineers firstly with their advantage of saving energy. However, the density of atmosphere can be so low in beginning of reentry flight that the desired control torque may be unachievable with the employment of aerodynamic surfaces alone because of poor aerodynamic maneuverability. As a result, RVs have to rely on reaction control system (RCS) jets in addition to aerodynamic surfaces. In this case, control allocation among redundant actuators becomes necessary, which further raises the difficulties in attitude control design. Meanwhile, a robust attitude control system for RVs with redundant actuators is desirable.
The conventional attitude control method for RVs is gain scheduling (GS) $[2,3]$. This method linearizes the system with a set of trimmed points, designs individual gains at each point, and then interpolates those gains online with respect to system parameters such as dynamic pressure or Mach number. Nevertheless, the conventional GS involves the lack of guaranteed global robustness and stability [4]. The reentry flight conditions change rapidly, which makes this method impractical [5]. Moreover, the point designs of gain scheduling are manpower intensive and highly time consuming [6]. To conclude, GS is weak at performing linearity.

Compared to GS, feedback linearization (FBL) [7-10] can exactly cancel the model nonlinearities and replace undesirable dynamics with desirable dynamic using nonlinear coordinate transformation. However, FBL relies on the knowledge of the exact model dynamics, which severely influences FBL's practicality because uncertainties and disturbances exist inevitably. To improve the flight control performance systematically on the basis of FBL, Rahideh et al. [7] 
incorporated neural network (NN) based compensation in the FBL design; Van Soest et al. [8] combined FBL with constrained linear model predictive control (MPC) method; Xu et al. [9] utilized the combination of FBL and adaptive sliding mode control (SMC) method.

Among the various upgraded nonlinear control methods, SMC outstands with many advantages, such as simplicity of implementation, fast dynamic response, good transient behavior, exponential stability, insensitivity to parameter variations, and robustness to plant uncertainties and external disturbances [11-14]. Therefore, SMC has been successfully applied to a variety of complex engineering systems [15]. Barambones Caramazana et al. [14] develop a sliding mode position control incorporating a flux estimator for highperformance real-time applications of induction motors. Wu et al. [15] investigate the key problems of SMC of Markovian jump singular time-delay systems. Shtessel et al. [16-19] studied the application of SMC method to reusable launch vehicle (RLV) in launch and reentry mode, and a multiple-time-scale SMC strategy is proposed in [18] for RLV in ascent phase.

Generally, SMC design consists of two steps [20, 21]: (1) select a sliding surface as a function of the system states so that the system trajectories along the surface meet the desired performance, such as stability and tracking capability; (2) design a suitable control law to drive the states onto the predefined sliding surface in finite time. When it comes to the design of conventional SMC, there are two major problems concerned. One is its unguaranteed global robustness and the other is chattering phenomenon. The conventional sliding surfaces [16-18] employ linear function of tracking errors, which results in the fact that the transient dynamics of SMC consists of reaching phase and sliding phase. However, the SMC method can only ensure the robustness against planet uncertainties and external disturbances in sliding phase. Therefore, the conventional sliding surfaces do not possess the property of global robustness. Several studies are dedicated to global robustness of SMC. Sun et al. [22] introduced an integral sliding mode control (ISMC) method to solve the longitudinal control problem of air-breathing hypersonic vehicle (AHV). Shtessel et al. [19] proposed a two-loop controller that utilized a time-varying sliding mode control (TVSMC) method to achieve fault tolerance for RLV attitude control. With the elimination of reaching phase, both ISMC and TVSMC can keep the system states on the sliding surface from the initial time, so that global robustness against planet uncertainties and external disturbances is guaranteed.

As to the chattering phenomenon, it is assumed that the control can be switched from one structure to another infinitely fast in the design of SMC [23]. However, it is impossible to achieve high-speed switching control because of the inevitable switching delay computation and the limitation of the physical actuators. The existence of time delay introduces instability, oscillation, and poor performance [24]. High control gains of SMC lead to high frequency oscillations known as chattering phenomenon. This harmful phenomenon may erode the performance to gain robustness, decrease the control accuracy, and damage the actuators [25]. There are essentially two ways to alleviate the chattering phenomenon [23]: one way is boundary layer method $[17-19,26]$ and the other is higher order sliding mode control (HOSMC). The boundary layer method replaces the sign function (discontinuous control) with smooth approximations, such as high-gain saturation function or sigmoid function. Nevertheless, this method no longer drives the system state to the origin and cannot guarantee the robustness and accuracy within the boundary layer [23]. HOSMC was proposed by Levant [27]. Instead of influencing the first order time derivative, the discontinuous control acts on sliding variable's higher order derivative. As a special case of HOSMC, second-order sliding mode control (SOSMC) is the most popular approach in engineering. There are many kinds of SOSMC, such as twisting algorithm [28], super-twisting algorithm [29], suboptimal algorithm [30, 31], and prescribed convergence law algorithm [28].

Disturbance observer (DO) is an effective way to enhance system robustness. The disturbance estimation is used for compensation. DO was first proposed by Ohishi et al. [32]. Hall and Shtessel [33] combined SMC and sliding mode disturbance observer (SMDO), which estimates the bounded uncertainties and disturbances effectively to improve RLV attitude control. Shtessel et al. [34] proposed a homogeneous DO based on the standard robust exact differentiator to solve the missile guidance problem.

Inspired by previous work, this paper proposes two TVSMC methods to solve the finite-time attitude control problem by incorporating the disturbance observer. One is BTVSMC/DO which is the abbreviation for boundary layer method based time-varying sliding mode controller with disturbance observer, and the other is SOTVSMC/DO which means the second-order time-varying sliding mode controller with disturbance observer. With the same dedication to systematically enhance robustness and suppress control chattering, the two methods adopt different ways to alleviate chattering. The former is designed based on boundary layer technique, and the latter utilizes a novel SOSMC. The main contributions of this paper are summarized as follows.

(1) This paper incorporates a novel reaching law based on SOSMC with the time-varying sliding function. In order to enhance the robustness of the method, a DO based on the standard robust exact differentiator is employed to estimate the system's uncertainties and disturbances in finite time. In addition, the finitetime convergence of time-varying sliding function for resulted method is proved via Lyapunov theory, and consequently the asymptotical stability of the closedloop nonlinear system is proved according to the definition of the time-varying sliding function.

(2) Since RVs deploy both aerodynamic surfaces and RCS jets, this paper introduces a control allocation approach to assign control responsibility amongst redundant actuators. The nonlinear programming problem is established and solved by optimization method, and the pulse-width-pulse-frequency (PWPF) is employed to modulate the on-off thrusters.

(3) The proposed control methods are applied to finitetime attitude control problem for RVs. Numerical simulation results confirm the validity and superior performance of the proposed control methods by 
comparing them with other conventional control methods. The comparison between boundary layer method and SOSMC is also presented.

The major contents of the following part in this paper are as follows. Section 2 describes the rotational equations of motion and formulates problems of attitude controller and control allocation. In Section 3, feedback linearization technique is employed to the equations of motion. Section 4 presents two TVSMC methods as well as the corresponding stability analysis. A control allocation method is introduced in Section 5. In Section 6, the performances of proposed control methods are assessed by numerical tests. Finally, Section 7 summarizes and lists the conclusions.

\section{Preliminary}

2.1. The Rotational Equations of Motion. Reentry guidance is concerned with steering the vehicle from entry interface (EI) to the designated target point in prescribed condition while satisfying necessary path constraints such as heating rate constraint, aerodynamic load constraint, and dynamic pressure constraint $[35,36]$. The steering commands are defined in terms of angle of attack (AOA) $\alpha_{c}$, sideslip angle $\beta_{c}$, and bank angle $\sigma_{c}$. Furthermore, to prevent excessive heat buildup, $\beta_{c}$ is kept around zero under the application of back-to-turn (BTT) control policy [37]. The subsequent reentry control system tracks these three attitude commands. And the objective of the reentry control system is to determine the actuator command vector $\boldsymbol{\delta}$ so that the reentry vehicle can follow the attitude commands that are specified by guidance system.

The motion of reentry vehicle can be divided into translational motion and rotational motion. Since the focus of this paper is about control system, the translational equations of motion utilized in guidance system are not presented. The reentry dynamics are governed by a group of nonlinear differential equations [38]. The kinematic equations of reentry vehicle are defined as [37]

$$
\begin{aligned}
\frac{d \alpha}{d t}= & -p \cos \alpha \tan \beta+q-r \sin \alpha \tan \beta \\
& +\frac{\sin \sigma}{\cos \beta}(\dot{\psi} \cos \gamma-\dot{\phi} \sin \psi \sin \gamma \\
& \left.+\left(\dot{\theta}+\omega_{e}\right)(\cos \phi \cos \psi \sin \gamma-\sin \phi \cos \gamma)\right) \\
& -\frac{\cos \sigma}{\cos \beta}\left(\dot{\gamma}-\dot{\phi} \cos \psi-\left(\dot{\theta}+\omega_{e}\right) \cos \phi \sin \psi\right), \\
\frac{d \beta}{d t}= & p \sin \alpha-r \cos \alpha \\
& +\sin \sigma\left(\dot{\gamma}-\dot{\phi} \cos \psi-\left(\dot{\theta}+\omega_{e}\right) \cos \phi \sin \psi\right) \\
& +\cos \sigma(\dot{\psi} \cos \gamma-\dot{\phi} \sin \psi \sin \gamma \\
& \left.\quad+\left(\dot{\theta}+\omega_{e}\right)(\cos \phi \cos \psi \sin \gamma-\sin \phi \cos \gamma)\right),
\end{aligned}
$$

$$
\begin{aligned}
\frac{d \sigma}{d t}= & -p \cos \alpha \cos \beta-q \sin \beta-r \sin \alpha \cos \beta \\
& +\dot{\alpha} \sin \beta-\dot{\psi} \sin \gamma-\dot{\phi} \sin \psi \cos \gamma \\
& +\left(\dot{\theta}+\omega_{e}\right)(\cos \phi \cos \psi \cos \gamma-\sin \phi \sin \gamma),
\end{aligned}
$$

where $\alpha, \beta$, and $\sigma$ are AOA, sideslip angle, and bank angle, respectively. $p, q$, and $r$ are the rates of roll, pitch, and yaw, respectively. $\gamma$ denotes flight path angle and $\psi$ denotes heading angle. $\theta$ and $\phi$ are longitude and latitude of reentry vehicle. $\omega_{e}$ is the angular rate of Earth rotation.

In order to simplify the online calculation, this paper obtains the kinetics of reentry vehicle under the following assumption.

Assumption 1. (1) The reentry vehicle is a rigid body; the terms impacted by elastic effects are not considered. (2) The reentry vehicle has a longitudinal symmetry plane, which means the products of inertia $I_{x y}=I_{y z}=0$. (3) The vehicle is unpowered during reentry. [37]

Hence, the kinetics of reentry vehicle can be expressed as

$$
\begin{aligned}
& \frac{\mathrm{d} p}{\mathrm{~d} t} \\
& =\frac{I_{z} M_{l}+I_{z x} M_{n}+I_{z x}\left(I_{z}+I_{x}-I_{y}\right) p q+\left(I_{y} I_{z}-I_{z}^{2}-I_{z x}^{2}\right) q r}{I_{x} I_{z}-I_{z x}^{2}}, \\
& \frac{\mathrm{d} q}{\mathrm{~d} t}=\frac{M_{m}+\left(I_{z}-I_{x}\right) p r+I_{z x}\left(r^{2}-p^{2}\right)}{I_{y}}, \\
& \frac{\mathrm{d} r}{\mathrm{~d} t} \\
& =\frac{I_{z x} M_{l}+I_{x} M_{n}+\left(-I_{x} I_{y}+I_{x}^{2}+I_{z x}^{2}\right) p q+I_{z x}\left(-I_{z}-I_{x}+I_{y}\right) q r}{I_{x} I_{z}-I_{z x}^{2}},
\end{aligned}
$$

where $M_{l}, M_{m}$, and $M_{n}$ are three control torques defined in the body frame roll pitch and yaw, respectively. $I_{x}, I_{y}$, and $I_{z}$ denote the moments of inertia, and $I_{z x}$ denotes the product of inertia.

The control-oriented model can be developed for control design based on (1) and (2). Since the rotational motions are much faster than translational motions and the motion of Earth, the translational terms and angular velocity of earth can be neglected; that is, $\dot{\gamma}=\dot{\psi}=\dot{\theta}=\dot{\phi}=0, \omega_{e}=0$. Therefore, the rotational equations of motions (1) and (2) can be further simplified as follows:

$$
\begin{aligned}
& \dot{\mathbf{\Omega}}=\mathbf{R} \boldsymbol{\omega}+\Delta \mathbf{f} \\
& \dot{\boldsymbol{\omega}}=-\mathbf{I}^{-1} \boldsymbol{\omega}^{\times} \mathbf{I} \boldsymbol{\omega}+\mathbf{I}^{-1} \mathbf{M}_{c}+\Delta \mathbf{d},
\end{aligned}
$$


where $\boldsymbol{\Omega}=[\alpha, \beta, \sigma]^{T}$ is the attitude angle vector, $\boldsymbol{\omega}=[p, q, r]^{T}$ is the attitude angular rate vector, and $\mathbf{M}_{c}=\left[M_{l}, M_{m}, M_{n}\right]^{T}$ is the command control torque vector. $\mathbf{R} \in \mathfrak{R}^{3 \times 3}$ is the coordinate-transformation matrix, $\Delta \mathbf{f}=\left[\Delta f_{1}, \Delta f_{2}, \Delta f_{3},\right]^{T}$ denotes the unknown bounded uncertainties caused by the model reduction, $\boldsymbol{\omega}^{\times} \in \mathfrak{R}^{3 \times 3}$ stands for the skew-symmetric matrix operator on vector $\boldsymbol{\omega}, \mathbf{I} \in \mathfrak{R}^{3 \times 3}$ denotes the symmetric positive definite inertia matrix of reentry vehicle, and $\Delta \mathbf{d} \epsilon$ $\Re^{3}$ denotes the bounded uncertain term. $\mathbf{R}, \boldsymbol{\omega}^{\times}, \mathbf{I}$, and $\Delta \mathbf{d}$ are given by

$$
\begin{aligned}
\mathbf{R} & =\left[\begin{array}{ccc}
-\cos \alpha \tan \beta & 1 & -\sin \alpha \tan \beta \\
\sin \alpha & 0 & -\cos \alpha \\
-\cos \alpha \cos \beta & -\sin \beta & -\sin \alpha \cos \beta
\end{array}\right], \\
\boldsymbol{\omega}^{\times} & =\left[\begin{array}{ccc}
0 & -r & q \\
r & 0 & -p \\
q & p & 0
\end{array}\right], \\
\mathbf{I} & =\left[\begin{array}{ccc}
I_{x} & 0 & -I_{z x} \\
0 & I_{y} & 0 \\
-I_{z x} & 0 & I_{z}
\end{array}\right], \\
\Delta \mathbf{d} & =\mathbf{I}^{-1}\left[-\Delta \mathbf{I} \dot{\boldsymbol{\omega}}-\boldsymbol{\omega}^{\times} \Delta \mathbf{I} \boldsymbol{\omega}+\Delta \mathbf{M}\right],
\end{aligned}
$$

where $\Delta \mathbf{I} \in \mathfrak{R}^{3 \times 3}$ denotes unknown bounded inertia variations and $\Delta \mathbf{M} \in \mathfrak{R}^{3}$ stands for the bounded external disturbance moment.

2.2. Problem Formulation. As shown in Figure 1, the control problem for reentry vehicle with redundant actuators can be solved in two steps. They are

(1) specifying the control torque vector $\mathbf{M}_{c}=\left[M_{l}, M_{m}\right.$, $\left.M_{n}\right]^{T}$ in equation set (3), which leads the output vector $\Omega$ to track the attitude command $\boldsymbol{\Omega}_{c}=\left[\alpha_{c}, \beta_{c}, \sigma_{c}\right]^{T}$ in a finite time:

$$
\lim _{t>t_{F}}\|\mathbf{e}\|=\lim _{t>t_{F}}\left\|\mathbf{\Omega}-\boldsymbol{\Omega}_{c}\right\|=0,
$$

where $\mathbf{e}=\mathbf{\Omega}-\boldsymbol{\Omega}_{c}$ is the tracking error;

(2) designing a control allocation method $f_{\mathrm{CA}}(\cdot)$ that maps the command control torque vector $\mathbf{M}_{c}$ to actuator deflection commands $\boldsymbol{\delta}_{c}$ [39]:

$$
\boldsymbol{\delta}_{c}=f_{\mathrm{CA}}\left(\mathbf{M}_{c}\right) .
$$

The actual torque produced by control allocation may not exactly equal the torque command. Assume that $\Delta \mathbf{M}_{c a}$ is the bounded disturbance caused by the process of control allocation, and the torque vector produced by actuators can be expressed as $\mathbf{M}_{c}+\Delta \mathbf{M}_{c a}$. Hence, the bounded uncertain term of (5) can be rewritten as

$$
\Delta \mathbf{d}=\mathbf{I}^{-1}\left[-\Delta \mathbf{I} \dot{\boldsymbol{\omega}}-\boldsymbol{\omega}^{\times} \Delta \mathbf{I} \boldsymbol{\omega}+\Delta \mathbf{M}+\Delta \mathbf{M}_{c a}\right] .
$$

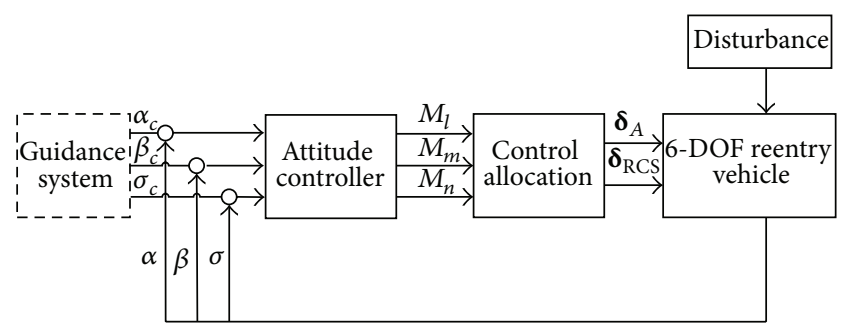

FIGURE 1: Control architecture for reentry vehicle.

\section{Feedback Linearization}

By the selection of control input as control torque vector $\mathbf{M}$ and the output as attitude angle vector $\boldsymbol{\Omega}$, the nonlinear attitude equations (3) can be expressed as [40]

$$
\begin{aligned}
& \dot{\mathbf{x}}=\mathbf{f}(\mathbf{x})+\mathbf{g}(\mathbf{x}) \mathbf{u}+\mathbf{d}, \\
& \mathbf{y}=\mathbf{h}(\mathbf{x}),
\end{aligned}
$$

where $\mathbf{x}=[\alpha, \beta, \sigma, p, q, r]^{T}$ is the state vector, $\mathbf{y}=[\alpha, \beta, \sigma]^{T}$ is the output vector, $\mathbf{u}=\left[M_{l}, M_{m}, M_{n}\right]^{T}$ is the control vector, and $\mathbf{d}=\left[\Delta \mathbf{f}^{T}, \Delta \mathbf{d}^{T}\right]^{T}$ stands for the system uncertain term. $\mathbf{f}(\mathbf{x})$ and $\mathbf{g}(\mathbf{x})$ can be obtained by (10) and (11), respectively:

$\mathbf{f}(\mathbf{x})=\left[\begin{array}{l}f_{1}(\mathbf{x}) \\ f_{2}(\mathbf{x}) \\ f_{3}(\mathbf{x}) \\ f_{4}(\mathbf{x}) \\ f_{5}(\mathbf{x}) \\ f_{6}(\mathbf{x})\end{array}\right]$

$$
=\left[\begin{array}{c}
-p \cos \alpha \tan \beta+q-r \sin \alpha \tan \beta \\
p \sin \alpha-r \cos \alpha \\
-p \cos \alpha \cos \beta-q \sin \beta-r \sin \alpha \cos \beta \\
\frac{I_{z x}\left(I_{z}+I_{x}-I_{y}\right) p q+\left(I_{y} I_{z}-I_{z}^{2}-I_{z x}^{2}\right) q r}{I_{x} I_{z}-I_{z x}^{2}} \\
\frac{\left(I_{z}-I_{x}\right) p r+I_{z x}\left(r^{2}-p^{2}\right)}{I_{y}} \\
\frac{\left(-I_{x} I_{y}+I_{x}^{2}+I_{z x}^{2}\right) p q+I_{z x}\left(-I_{z}-I_{x}+I_{y}\right) q r}{I_{x} I_{z}-I_{z x}^{2}}
\end{array}\right],
$$

$$
\begin{aligned}
\mathbf{g}(\mathbf{x}) & =\left[\begin{array}{lll}
g_{1}(\mathbf{x}) & g_{2}(\mathbf{x}) & g_{3}(\mathbf{x})
\end{array}\right] \\
& =\left[\begin{array}{ccc}
0 & 0 & 0 \\
0 & 0 & 0 \\
0 & 0 & 0 \\
\frac{I_{z}}{I_{x} I_{z}-I_{z x}^{2}} & 0 & \frac{I_{z x}}{I_{x} I_{z}-I_{z x}^{2}} \\
0 & \frac{1}{I_{y}} & 0 \\
\frac{I_{z x}}{I_{x} I_{z}-I_{z x}^{2}} & 0 & \frac{I_{x}}{I_{x} I_{z}-I_{z x}^{2}}
\end{array}\right] .
\end{aligned}
$$


The vector relative degree of system $(9)$ is $(2,2,2)$. After differentiating output vector $\mathbf{y}$ twice, the control input vector u appears:

$$
\ddot{\mathbf{y}}=\mathbf{K u}+\mathbf{B}+\mathbf{R I}^{-1} \Delta \mathbf{d}+\mathbf{F} \Delta \mathbf{f},
$$

where $\mathbf{K}, \mathbf{B}$, and $\mathbf{F}$ are given by

$$
\begin{aligned}
\mathbf{K} & =\left[\begin{array}{lll}
L_{g_{1}} L_{f} h_{1}(\mathbf{x}) & L_{g_{2}} L_{f} h_{1}(\mathbf{x}) & L_{g_{3}} L_{f} h_{1}(\mathbf{x}) \\
L_{g_{1}} L_{f} h_{2}(\mathbf{x}) & L_{g_{2}} L_{f} h_{2}(\mathbf{x}) & L_{g_{3}} L_{f} h_{2}(\mathbf{x}) \\
L_{g_{1}} L_{f} h_{3}(\mathbf{x}) & L_{g_{2}} L_{f} h_{3}(\mathbf{x}) & L_{g_{3}} L_{f} h_{3}(\mathbf{x})
\end{array}\right], \\
\mathbf{B} & =\left[\begin{array}{lll}
\left.L_{f}^{2} h_{1}(\mathbf{x}), L_{f}^{2} h_{2}(\mathbf{x}), L_{f}^{2} h_{3}(\mathbf{x})\right]^{T}, \\
\mathbf{F}=\left[\begin{array}{ccc}
p \sin \alpha \tan \beta-r \cos \alpha \tan \beta+1 & -p \cos \alpha \sec ^{2} \beta-r \sin \alpha \sec ^{2} \beta & 0 \\
p \cos \alpha+r \cos \alpha & 1 & 0 \\
p \sin \alpha \cos \beta-r \cos \alpha \cos \beta & p \cos \alpha \sin \beta-q \cos \beta+r \sin \alpha \sin \beta & 1
\end{array}\right] .
\end{array}\right.
\end{aligned}
$$

According to (12), the total relative degree of system equals the order of the system. Furthermore, since the sideslip angle $\beta \approx 0$ during reentry,

$$
\operatorname{det}(\mathbf{K})=\frac{\cos \beta-\sin \beta \tan \beta}{I_{x} I_{z}-I_{z x}^{2}} \approx \frac{1}{I_{x} I_{z}-I_{z x}^{2}} \neq 0 .
$$

Thus, the system (9) can be linearized completely without zero dynamics by using the following feedback control law:

$$
\mathbf{u}=\mathbf{K}^{-1}(-\mathbf{B}+\mathbf{v})
$$

where $\mathbf{v}=\left[v_{1}, v_{2}, v_{3}\right]^{T}$ is selected as the new control input in this paper.

Define the bounded uncertainty terms $\Delta \mathbf{v}=\mathbf{R I}^{-\mathbf{1}} \Delta \mathbf{d}+$ $\mathbf{F} \Delta \mathbf{f}$ as the lumped uncertainty, and substitute (15) into (12), and the basic model for attitude controller design can be obtained by

$$
\ddot{\mathbf{y}}=\mathbf{v}+\Delta \mathbf{v}
$$

\section{Sliding Mode Attitude Controller Design}

This section develops two TVSMC attitude controllers to solve the finite-time control problem by incorporating disturbance observer. The first controller is BTVSMC/DO and the second controller is SOTVSMC/DO. This part elaborates on the design of sliding surface and reaching law of the controllers. Moreover, the design of disturbance observer is presented, too.

4.1. Time-Varying Sliding Surface Design. The time-varying sliding surface is selected as [41]

$$
\mathbf{s}=\dot{\mathbf{e}}+\mathbf{c e}+\mathbf{a} \mathbf{e}^{-\lambda t},
$$

where $\mathbf{s}=\left[s_{1}, s_{2}, s_{3}\right]^{T}$, the tracking error vector $\mathbf{e}=\mathbf{y}-\mathbf{y}_{\mathbf{c}}=$ $\left[e_{1}, e_{2}, e_{3}\right]^{T}, \mathbf{c}=\operatorname{diag}\left(c_{1}, c_{2}, c_{3}\right)$ is the sliding function gain matrix, and the element $c_{i}>0, i=1,2,3, \lambda \in \mathfrak{R}^{+}, \mathbf{a}=$ $\left[a_{1}, a_{2}, a_{3}\right]^{T}$ is the coefficient vector to guarantee the existence of sliding mode from the beginning of motion. Hence, $\mathbf{a}$ is defined as

$$
\mathbf{a}=-\dot{\mathbf{e}}(0)-\mathbf{c e}(0) .
$$

Lemma 2. If the sliding mode $\mathbf{s}(t)=\mathbf{0}_{3 \times 1}$ is satisfied, the system (9) is globally asymptotically stable.

Proof. According to (17), $\mathbf{s}(t)=\mathbf{0}_{3 \times 1}$ can be rewritten in scalar form:

$$
\dot{e}_{i}+c_{i} e_{i}+a_{i} e^{-\lambda t}=0, \quad i=1,2,3 .
$$

If $c_{i} \neq \lambda$, the differential equations can be solved as

$$
\begin{aligned}
e_{i}(t) & =\frac{c_{i} e_{i}(0)}{c_{i}-\lambda} e^{-\lambda t}-\frac{\lambda e_{i}(0)}{c_{i}-\lambda} e^{-c_{i} t} \\
& \leq\left|\frac{c_{i} e_{i}(0)}{c_{i}-\lambda}-\frac{\lambda e_{i}(0)}{c_{i}-\lambda}\right| e^{-\varsigma t},
\end{aligned}
$$

where $\varsigma=\min \left(c_{i}, \lambda\right)$, and thus the system (9) is globally exponentially stable.

If $c_{i}=\lambda$, the differential equation can be solved as

$$
e_{i}(t)=e^{-c_{i} t}\left(c_{i} t+1\right) e_{i}(0) \text {. }
$$

Because $\lim _{t \rightarrow \infty} e_{i}(t) \rightarrow 0$, the system (9) is globally asymptotically stable.

In conclusion, the asymptotic stability of the system (9) is guaranteed when sliding mode $\mathbf{s}(t)=\mathbf{0}_{3 \times 1}$ is satisfied. This completes the proof.

Remark 3. To simplify the selection procedure, the four parameters $c_{1}, c_{2}, c_{3}$, and $\lambda$ in (17) are set equal so that each of them is able to determine the sliding surface. As the parameters become larger, the rate of tracking error is faster and the control input is required to be larger. However, control input in real situation could not always be bigger as a faster convergence rate requires. As a result, a tradeoff between control input and convergence rate is necessary, which can be achieved by trial-and-error method. 
4.2. Disturbance Observer Design. The first order derivative of the sliding surface $\mathbf{s}$ is

$$
\begin{aligned}
\dot{\mathbf{s}} & =\ddot{\mathbf{e}}+\mathbf{c} \dot{\mathbf{e}}-\lambda \mathbf{a} \mathbf{e}^{-\lambda t} \\
& =-\ddot{\mathbf{y}}_{\mathbf{c}}+\ddot{\mathbf{y}}+\mathbf{c} \dot{\mathbf{e}}-\lambda \mathbf{a} \mathbf{e}^{-\lambda t} \\
& =-\mathbf{b}+\mathbf{v}+\Delta \mathbf{v},
\end{aligned}
$$

where $\mathbf{b}=\ddot{\mathbf{y}}_{\mathbf{c}}-\mathbf{c} \dot{\mathbf{e}}+\lambda \mathbf{a} \mathbf{e}^{-\lambda t}$.

Hence, the control vector $\mathbf{v}$ can be expressed as

$$
\mathbf{v}=\mathbf{b}-\Delta \mathbf{v}+\dot{\mathbf{s}} .
$$

The sliding variable dynamics (22) is sensitive to the unknown bounded term $\Delta \mathbf{v}$. However, the detailed information of $\Delta \mathbf{v}$ in (23) is unavailable. To estimate the lumped uncertainty, the robust differentiator technique [34] is employed.

Assumption 4. $s_{1}, s_{2}, s_{3}$ are measured by Lebesgue-measurable noise bounded $\varepsilon_{1}>0, \varepsilon_{2}>0, \varepsilon_{3}>0$, respectively. Furthermore, $\mathbf{v}, \mathbf{b}$ are assumed to be bounded and Lebesgue measurable respectively, and the lumped uncertainty $\Delta \mathbf{v}$ is 2 times differentiable and bounded.

Consider $\mathbf{z}_{0}=\left[z_{01}, z_{02}, z_{03}\right]^{T}, \mathbf{z}_{1}=\left[z_{11}, z_{12}, z_{13}\right]^{T}$, and $\mathbf{z}_{2}=\left[z_{21}, z_{22}, z_{23}\right]^{T}$ as the estimated values of state variables, and the observer can be expressed as [34]

$$
\begin{gathered}
\dot{\mathbf{z}}_{0}=\mathbf{v}-\mathbf{b}+\boldsymbol{v}_{0} \\
\boldsymbol{v}_{0}=-\lambda_{0} \mathbf{L}^{1 / 3}\left[\begin{array}{ccc}
\left|z_{01}-s_{1}\right|^{2 / 3} & 0 & 0 \\
0 & \left|z_{02}-s_{2}\right|^{2 / 3} & 0 \\
0 & 0 & \left|z_{03}-s_{3}\right|^{2 / 3}
\end{array}\right] \\
\times \operatorname{sgn}\left(\mathbf{z}_{0}-\mathbf{s}\right)+\mathbf{z}_{1}, \\
\boldsymbol{v}_{1}=-\lambda_{1} \mathbf{L}^{1 / 2}\left[\begin{array}{cc}
\left|z_{11}-v_{01}\right|^{1 / 2} \\
0 \\
0 \\
\dot{\mathbf{z}}_{1}=\boldsymbol{v}_{1}, \\
\quad\left|z_{12}-v_{02}\right|^{1 / 2} & 0 \\
0 & \left|z_{13}-v_{03}\right|^{1 / 2}
\end{array}\right] \\
\dot{\mathbf{z}}_{2}=-\lambda_{2} \mathbf{L} \operatorname{sgn}\left(\mathbf{z}_{2}-v_{1}\right),
\end{gathered}
$$

where $\boldsymbol{v}_{0}=\left[v_{01}, v_{02}, v_{03}\right]^{T}, \boldsymbol{\nu}_{0}=\left[\nu_{01}, \nu_{02}, v_{03}\right]^{T} . \lambda_{0}, \lambda_{1}, \lambda_{2}$ and $\mathbf{L}=\operatorname{diag}\left(l_{1}, l_{2}, l_{3}\right)$ are the parameters to be selected.
Lemma 5 (see [34]). Suppose Assumption 4 is satisfied. DO (24) is finite-time stable. The following inequalities can be established in finite time:

$$
\begin{array}{r}
z_{0 i}-s_{i} \leq \mu_{0 i} \varepsilon_{i}, \\
z_{1 i}-\Delta v_{i} \leq \mu_{1 i} \varepsilon_{i}^{2 / 3}, \\
z_{2 i}-\Delta \dot{v}_{i} \leq \mu_{2 i} \varepsilon_{i}^{1 / 3}, \\
i=1,2,3,
\end{array}
$$

where $\mu_{0 i}, \mu_{1 i}, \mu_{2 i}, i=1,2,3$, are positive constants.

Remark 6. The proof of Lemma 5 is similar to the studies of Shtessel et al. [34] and is not presented in this paper. The parameters $\lambda_{0}, \lambda_{1}, \lambda_{2}$ can be chosen recursively, and the simulation-checked set $8,5,3$ is suitable for the observer design $[34,42]$.

In absence of measurement noise, the exact equalities can be established in a finite time:

$$
\mathrm{z}_{0}=\mathrm{s}, \quad \mathrm{z}_{1}=\Delta \mathrm{v}, \quad \mathrm{z}_{2}=\Delta \dot{\mathrm{v}}
$$

After DO is constructed, the control vector $\mathbf{v}$ can be modified as

$$
\mathbf{v}=\mathbf{b}-\mathbf{z}_{1}+\dot{\mathbf{s}} .
$$

4.3. Reaching Law Design. Before giving the reaching law design, three lemmas to be used are presented.

Lemma 7 (see [43]). Consider the system of differential equations:

$$
\dot{x}(t)=f(x(t)),
$$

where $x \in \Re^{n}, f: D_{0} \rightarrow \Re^{n}$ is continuous on an open neighborhood $D_{0}$ containing the origin, $f(0)=0$.

Suppose there exists a continuous positive definite function $V: D_{0} \rightarrow \Re$. In addition, there exist real numbers $k>0$, $\alpha \in(0,1)$, and an open neighborhood of the origin $D_{1} \subseteq D_{0}$ satisfies

$$
\dot{V}+k V^{\alpha} \leq 0, \quad x \in D_{1} \backslash\{0\} .
$$

Then the origin is a finite-time stable equilibrium of system (28). The settling time $t$ is depended on the initial value $x_{0}$ :

$$
t\left(x_{0}\right) \leq \frac{V\left(x_{0}\right)^{1-\alpha}}{k(1-\alpha)} .
$$

Furthermore, if $D=D_{0}=\mathfrak{R}^{n}$, the origin is a globally finitetime stable equilibrium of system (28).

Lemma 8 (see [44]). Suppose there exists a continuous positive definite function $V: D_{0} \rightarrow \mathfrak{R}$. In addition, there exist real numbers $k, l>0, \alpha \in(0,1)$, and an open neighborhood of the origin $D_{1} \subseteq D_{0}$ satisfies

$$
\dot{V}+k V^{\alpha}+l V \leq 0, \quad x \in D_{1} \backslash\{0\} .
$$


Then the origin is a finite-time stable equilibrium of system (28). The settling time $t$ is depended on the initial value $x_{0}$ :

$$
t\left(x_{0}\right) \leq \frac{\ln \left(1+(l / k) V\left(x_{0}\right)^{1-\alpha}\right)}{k(1-\alpha)} .
$$

Furthermore, if $D=D_{0}=\Re^{n}$, the origin is a globally finitetime stable equilibrium of system (28).

Lemma 9 (see [45]). For $x_{i} \in \mathfrak{R}, i=1, \ldots, n, 0<p \leq 1$ is a real number, and the inequality holds:

$$
\left(\left|x_{1}\right|+\cdots+\left|x_{n}\right|\right)^{p} \leq\left|x_{1}\right|^{p}+\cdots+\left|x_{n}\right|^{p} .
$$

Consider the reaching law with saturation function:

$$
\dot{\mathbf{s}}=-\boldsymbol{\eta} \text { sat }(\mathbf{s}),
$$

where $\boldsymbol{\eta}=\operatorname{diag}\left(\eta_{1}, \eta_{2}, \eta_{3}\right), \eta_{i} \in \mathfrak{R}^{+}, i=1,2,3$, sat $(\mathbf{s})=$ $\left[\operatorname{sat}\left(s_{1}\right), \operatorname{sat}\left(s_{2}\right), \operatorname{sat}\left(s_{3}\right)\right]^{T}$ stands for the saturation function that is used to attenuate the chattering problem, and $\operatorname{sat}\left(s_{i}\right)$, $i=1,2,3$, is defined as

$$
\operatorname{sat}\left(s_{i}\right)=\left\{\begin{array}{ll}
h_{i}^{-1} s_{i} & \left|s_{i}\right| \leq h_{i} \\
\operatorname{sgn}\left(s_{i}\right) & \left|s_{i}\right|>h_{i}
\end{array} \quad i=1,2,3,\right.
$$

where $\mathbf{h}=\operatorname{diag}\left(h_{1}, h_{2}, h_{3}\right)$ and $h_{i}$ is the boundary layer thickness.

Substitute (34) into (27); the control algorithm of BTVS$\mathrm{MC} / \mathrm{DO}$ can be expressed as

$$
\mathbf{v}=\mathbf{b}-\mathbf{z}_{1}-\boldsymbol{\eta} \text { sat }(\mathbf{s}) .
$$

Theorem 10. Based on Assumption 4, the attitude control problem described in (9) can be solved by BTVSMC/DO (36). Furthermore, the attitude tracking error $\mathbf{e}$ is asymptotically stable if the exact estimate of $\Delta \mathbf{v}$ is available through the DO.

Proof. Consider the Lyapunov function candidate:

$$
V_{1}=\frac{1}{2} \mathbf{s}^{T} \mathbf{s} .
$$

According to (37) and (22), the time derivative of $V_{1}$ is

$$
\begin{aligned}
\dot{V}_{1} & =\mathbf{s}^{T} \dot{\mathbf{s}} \\
& =\mathbf{s}^{T}(-\mathbf{b}+\mathbf{v}+\Delta \mathbf{v}) .
\end{aligned}
$$

Substituting (36) into (38) gives

$$
\dot{V}_{1}=\mathbf{s}^{T}\left(-\boldsymbol{\eta} \text { sat }(s)-\mathbf{z}_{1}+\Delta \mathbf{v}\right) .
$$

According to Lemma 5, DO (24) is finite-time stable; hence, we suppose there exists a moment $t=t_{\mathrm{ob}}$, which satisfies $\mathbf{z}_{1}=\Delta \mathbf{v}, t \geq t_{\mathrm{ob}}$.

When $t \geq t_{\mathrm{ob}}$,

$$
\begin{aligned}
\dot{V}_{1} & =\boldsymbol{s}^{T}(-\boldsymbol{\eta} \text { sat }(\mathbf{s})) \\
& =\sum_{i=1}^{3} \eta_{i} s_{i} \operatorname{sat}\left(s_{i}\right) .
\end{aligned}
$$

In view of (35), consider the following two cases.

(1) If $\left|s_{i}\right|>h_{i}, i=1,2,3$, we can get $\eta_{i} s_{i} \operatorname{sat}\left(s_{i}\right)=$ $\eta_{i} s_{i} \operatorname{sgn}\left(s_{i}\right)=\eta_{i}\left|s_{i}\right|$.

(2) If $\left|s_{i}\right| \leq h_{i}, i=1,2,3$, we can get $\eta_{i} s_{i} \operatorname{sat}\left(s_{i}\right)=$ $\eta_{i} s_{i} h_{i}^{-1} s_{i}=\eta_{i}\left|s_{i}\right|^{2} h_{i}^{-1} \leq \eta_{i}\left|s_{i}\right|$.

Hence, it is obvious that

$$
\begin{aligned}
\dot{V}_{1} & \leq \sum_{i=1}^{3} \eta_{i}\left|s_{i}\right| \\
& \leq-\chi \sqrt{V_{1}},
\end{aligned}
$$

where $\chi=\min \left(\eta_{1}, \eta_{2}, \eta_{3}\right)$; according to Lemma 7 , the trajectory of system will be driven into the related sliding surface $\mathbf{s}=\mathbf{0}_{3 \times 1}$ in a finite time $t_{r}$ :

$$
t_{r} \leq \frac{2 \sqrt{V_{1}\left(t_{\mathrm{ob}}\right)}}{\chi}+t_{\mathrm{ob}}
$$

where $V_{1}\left(t_{\mathrm{ob}}\right)$ is the value of $V_{1}$ at $t=t_{\mathrm{ob}}$.

According to Lemma 2, once the slide mode $\mathbf{s}=\mathbf{0}_{3 \times 1}$ is established, the system (9) is globally asymptotically stable. This completes the proof.

Generally, a thicker boundary layer (larger values of $h_{i}$, $i=1,2,3)$ contributes to smaller chattering; however, the static error inside the boundary layer may be large. Since the boundary layer method may result in the erosion of robustness and precision, a novel second-order SMC is pro-posed in this paper.

Consider the reaching law:

$$
\begin{aligned}
& \dot{\mathbf{s}}=-\mathbf{k}_{1} \operatorname{sig}^{(m-1) / m}(\mathbf{s}) \operatorname{sgn}(\mathbf{s})-\mathbf{k}_{2} \mathbf{s}+\boldsymbol{\tau}, \\
& \dot{\boldsymbol{\tau}}=\mathbf{k}_{3} \operatorname{sig}^{(m-2) / m}(\mathbf{s}) \operatorname{sgn}(\mathbf{s})-\mathbf{k}_{4} \mathbf{s},
\end{aligned}
$$

where $m>2, \mathbf{k}_{1}=\operatorname{diag}\left(k_{11}, k_{12}, k_{13}\right), \mathbf{k}_{2}=\operatorname{diag}\left(k_{21}, k_{22}, k_{23}\right)$, $\mathbf{k}_{3}=\operatorname{diag}\left(k_{31}, k_{32}, k_{33}\right)$, and $\mathbf{k}_{4}=\operatorname{diag}\left(k_{41}, k_{42}, k_{43}\right)$ with $k_{1 i}$, $k_{2 i}, k_{3 i}, k_{4 i} \in \mathfrak{R}^{+}, i=1,2,3$. And $\operatorname{sig}^{m}(\mathbf{s})$ is defined as

$$
\operatorname{sig}^{m}(\mathbf{s})=\left[\operatorname{sgn}\left(s_{1}\right)\left|s_{1}\right|^{m}, \operatorname{sgn}\left(s_{2}\right)\left|s_{2}\right|^{m}, \operatorname{sgn}\left(s_{3}\right)\left|s_{3}\right|^{m}\right]^{T} .
$$

Substitute (43) into (27); the related control algorithm of SOTVSMC/DO is given by

$$
\begin{aligned}
\mathbf{v}= & \mathbf{b}-\mathbf{z}_{1}-\mathbf{k}_{1} \operatorname{sig}^{(m-1) / m}(\mathbf{s}) \operatorname{sgn}(\mathbf{s})-\mathbf{k}_{2} \mathbf{s} \\
& +\int\left(\mathbf{k}_{3} \operatorname{sig}^{(m-2) / m}(\mathbf{s}) \operatorname{sgn}(\mathbf{s})-\mathbf{k}_{4} \mathbf{s}\right) .
\end{aligned}
$$

Remark 11. In view of (43), when the system's initial state is close to equilibrium point $\left(\mathbf{s}_{i}=0, i=1,2,3\right)$, the convergence speed mainly depends on the nonlinear terms. Otherwise, when the system's initial state is far from equilibrium 
point, the convergence speed mainly depends on the linear terms. Hence, the system can hold a fast convergence speed whether the initial state is close to equilibrium point or not.

Theorem 12. Suppose that the following inequality holds:

$$
\begin{array}{r}
m^{2} k_{3 i} k_{4 i}-\left(\frac{m^{3}}{m-1} k_{3 i}+\left(4 m^{2}-4 m+1\right) k_{1 i}^{2}\right) k_{2 i}^{2}>0 \\
i=1,2,3
\end{array}
$$

Based on Assumption 4, the attitude control problem described in (9) can be solved by the SOTVSMC/DO (45). Furthermore, the attitude tracking error $\mathbf{e}$ is asymptotically stable if the exact estimate of $\Delta \mathbf{v}$ is available through the DO.

Proof. The Lyapunov function can be expressed as

$$
V_{2}=\sum_{i=1}^{3} V_{2 i}, \quad i=1,2,3 .
$$

According to [46], $V_{2 i}$ is defined as

$$
\begin{aligned}
V_{2 i}= & \frac{1}{2}\left(-k_{1 i}\left|s_{i}\right|^{(m-1) / m} \operatorname{sgn}\left(s_{i}\right)-k_{2 i} s_{i}+\tau_{i}\right)^{2} \\
& +\frac{k_{3 i} m}{m-1}\left|s_{i}\right|^{2(m-1) / m}+k_{4 i} s_{i}^{2}+\frac{1}{2} \tau_{i}^{2} .
\end{aligned}
$$

Construct the vector $\boldsymbol{\xi}_{i}=\left[\left|s_{i}\right|^{(m-1) / m} \operatorname{sgn}\left(s_{i}\right), s_{i}, \tau_{i}\right]^{T}$; then $V_{2 i}$ and the derivative $\dot{V}_{2 i}$ can be expressed as

$$
\begin{aligned}
& V_{2 i}=\boldsymbol{\xi}_{i}^{T} \boldsymbol{\Lambda}_{i} \boldsymbol{\xi}_{i}, \\
& \dot{V}_{2 i}=-\left|s_{i}\right|^{-1 / m} \boldsymbol{\xi}_{i}^{T} \boldsymbol{\Gamma}_{1 i} \xi_{i}+\boldsymbol{\xi}_{i}^{T} \boldsymbol{\Gamma}_{2 i} \boldsymbol{\xi}_{i},
\end{aligned}
$$

where

$$
\Lambda_{i}=\frac{1}{2}\left[\begin{array}{ccc}
(2 m /(m-1)) k_{3 i}+k_{1 i}^{2} & k_{1 i} k_{2 i} & -k_{1 i} \\
k_{1 i} k_{2 i} & 2 k_{4 i}+k_{2 i}^{2} & -k_{2 i} \\
-k_{1 i} & -k_{2 i} & 2
\end{array}\right],
$$

$\Gamma_{1 i}$

$$
\begin{gathered}
=\frac{k_{1 i}}{m} \\
\times\left[\begin{array}{ccc}
m k_{3 i}+(m-1) k_{1 i}^{2} & 0 & -(m-1) k_{1 i} \\
0 & m k_{4 i}+(3 m-1) k_{2 i}^{2} & -(2 m-1) k_{2 i} \\
-(m-1) k_{1 i} & -(2 m-1) k_{2 i} & m-1
\end{array}\right], \\
\quad \Gamma_{2 i}=k_{2 i}\left[\begin{array}{ccc}
k_{3 i}+((3 m-2) / m) k_{1 i}^{2} & 0 & 0 \\
0 & k_{4 i}+k_{2 i}^{2} & -k_{2 i} \\
0 & -k_{2 i} & 1
\end{array}\right] .
\end{gathered}
$$

$V_{2 i}$ is continuous positive function:

$$
\lambda_{\min }\left\{\boldsymbol{\Lambda}_{i}\right\}\left\|\boldsymbol{\xi}_{i}\right\|^{2} \leq V_{2 i} \leq \lambda_{\max }\left\{\boldsymbol{\Lambda}_{i}\right\}\left\|\boldsymbol{\xi}_{i}\right\|^{2},
$$

where $\|\cdot\|$ denotes the Euclidean norm. $\lambda_{\min }\{\cdot\}$ and $\lambda_{\max }\{\cdot\}$ denote the minimum and maximum eigenvalues of the related matrix, respectively:

$$
\begin{gathered}
\left|s_{i}\right|^{(m-1) / m} \leq \sqrt{\left|s_{i}\right|^{2(m-1) / m}+s_{i}^{2}+\tau_{i}^{2}}=\left\|\boldsymbol{\xi}_{i}\right\| \leq \sqrt{\frac{V_{2 i}}{\lambda_{\min }\left\{\boldsymbol{\Lambda}_{i}\right\}}} \\
\left|s_{i}\right|^{1 / m} \leq\left(\frac{V_{2 i}}{\lambda_{\min }\left\{\boldsymbol{\Lambda}_{i}\right\}}\right)^{1 / 2(m-1)} .
\end{gathered}
$$

According to (46), $\Gamma_{1 i}$ and $\Gamma_{2 i}$ are positive definite matrixes; hence, $\dot{V}_{2 i}$ is negative:

$$
\begin{aligned}
\dot{V}_{2 i} \leq & -\left|s_{i}\right|^{-1 / m} \lambda_{\min }\left\{\boldsymbol{\Gamma}_{1 i}\right\}\left\|\boldsymbol{\xi}_{i}\right\|^{2}-\lambda_{\min }\left\{\boldsymbol{\Gamma}_{2 i}\right\}\left\|\boldsymbol{\xi}_{i}\right\|^{2} \\
\leq & -\left(\frac{\lambda_{\min }\left\{\boldsymbol{\Lambda}_{i}\right\}}{V_{2 i}}\right)^{1 / 2(m-1)} \\
& \times \lambda_{\min }\left\{\boldsymbol{\Gamma}_{1 i}\right\} \frac{V_{2 i}}{\lambda_{\max }\left\{\boldsymbol{\Lambda}_{i}\right\}}-\lambda_{\min }\left\{\boldsymbol{\Gamma}_{2 i}\right\} \frac{V_{2 i}}{\lambda_{\max }\left\{\boldsymbol{\Lambda}_{i}\right\}} \\
= & -\chi_{1 i} V_{2 i}^{(2 m-3) /(2 m-2)}-\chi_{2 i} V_{2 i},
\end{aligned}
$$

where $\chi_{1 i}=\left(\lambda_{\min }\left\{\boldsymbol{\Lambda}_{i}\right\}\right)^{1 / 2(m-1)}\left(\lambda_{\min }\left\{\boldsymbol{\Gamma}_{1 i}\right\} / \lambda_{\max }\left\{\boldsymbol{\Lambda}_{i}\right\}\right), \chi_{2 i}=$ $\left(\lambda_{\min }\left\{\boldsymbol{\Gamma}_{2 i}\right\} / \lambda_{\max }\left\{\boldsymbol{\Lambda}_{i}\right\}\right)$,

$$
\begin{aligned}
V_{2} & =\sum_{i=1}^{3}\left(-\chi_{1 i} V_{2 i}^{(2 m-3) /(2 m-2)}-\chi_{2 i} V_{2 i}\right) \\
& \leq-\chi_{1} \sum_{i=1}^{3} V_{2 i}^{(2 m-3) /(2 m-2)}-\chi_{2} V_{2},
\end{aligned}
$$

where $\chi_{1}=\max \left\{\chi_{11}, \chi_{12}, \chi_{13}\right\}, \chi_{2}=\max \left\{\chi_{21}, \chi_{22}, \chi_{23}\right\}$.

Since $m>2,0<(2 m-3) /(2 m-2)<1$. According to Lemma 9

$$
V_{2}+\chi_{1} V_{2}^{(2 m-3) /(2 m-2)}+\chi_{2} V_{2} \leq 0
$$

Similar to the proof of Theorem 10, according to Lemma 8 , the trajectory of system will be driven into the related sliding surface $\mathbf{s}=\mathbf{0}_{3 \times 1}$ in a finite time $t_{r}$ :

$$
t_{r} \leq \frac{2(m-1) \ln \left(1+\left(\chi_{2} / \chi_{1}\right) V_{2}\left(t_{\mathrm{ob}}\right)^{1 / 2(m-1)}\right)}{\chi_{1}}+t_{\mathrm{ob}}
$$

where $t_{\mathrm{ob}}$ is the moment which satisfies $\mathbf{z}_{1}=\Delta \mathbf{v}, t \geq t_{\mathrm{ob}}$. $V_{2}\left(t_{\mathrm{ob}}\right)$ is the value of $V_{2}$ at $t=t_{\mathrm{ob}}$.

According to Lemma 2, once the slide mode $\mathbf{s}=\mathbf{0}_{3 \times 1}$ is established, the system (9) is globally asymptotically stable. This completes the proof. 


\section{Control Allocation}

With low atmosphere density, reentry vehicle suffers poor aerodynamic maneuverability at high altitude. In such case, the combination of RCS jets and aerodynamic surfaces is considered to meet the control performances. To ensure that the command control torque $\mathbf{M}_{c}$ can be produced jointly by the actuators input $\boldsymbol{\delta}_{c}$, a control allocation approach is designed in this paper.

The core of the control allocation problem is to solve the nondeterministic system equations with typical constraints. Suppose that the number of aerodynamic surface is $n_{1}$, and the number of RCS jets is $n_{2}$ :

$$
\mathbf{M}_{c}=\mathbf{D}(\cdot) \boldsymbol{\delta}_{c},
$$

where $\boldsymbol{\delta}_{c}=\left[\boldsymbol{\delta}_{A} ; \boldsymbol{\delta}_{\mathrm{RCS}}\right], \boldsymbol{\delta}_{A}=\left[\delta_{A 1}, \delta_{A 2}, \ldots, \delta_{A n_{1}}\right]^{T}$ denotes the vector of aerodynamic surface deflection, and $\boldsymbol{\delta}_{\mathrm{RCS}}=$ $\left[\delta_{\mathrm{RCS} 1}, \delta_{\mathrm{RCS} 2}, \ldots, \delta_{\mathrm{RCSn} n_{2}}\right]$ stands for the vector of RCS thruster states. The matrix $\mathbf{D}(\cdot)$ can be expressed as

$$
\mathbf{D}(\cdot)=\left[\mathbf{D}_{A}(\cdot), \mathbf{D}_{\mathrm{RCS}}(\cdot)\right],
$$

where $\mathbf{D}_{A}(\cdot)$ and $\mathbf{D}_{\mathrm{RCS}}(\cdot)$ stand for aerodynamic torque matrix and RCS torque matrix, respectively.

The typical constraints for the control allocation problem are commonly defined as

$$
\begin{aligned}
\delta_{A i \min } & \leq \delta_{A i} \leq \delta_{A i \max }, \\
\delta_{d-A i \min } & \leq \dot{\delta}_{A i} \leq \delta_{d-A i \max }, \quad i=1, \ldots, n_{1}, \\
0 & \leq \delta_{\mathrm{RCS} j} \leq 1, \quad j=1, \ldots, n_{2},
\end{aligned}
$$

where $\delta_{A i \text { min }}$ and $\delta_{A i \text { max }}$ are the lower boundary and upper boundary of aerodynamic surface $\delta_{A i}$, respectively. $\delta_{d-A i \text { min }}$ and $\delta_{d-A i \max }$ are the lower boundary and upper boundary of deflection rate $\dot{\delta}_{A i}$, respectively.

The optimization method can be used to solve the nondeterministic system equations. The primary object of the control allocation is to minimize the difference between command control torque $\mathbf{M}_{c}$ and the torque produced by actuators [39]. Moreover, another objective is to minimize the use of RCS jets. Hence, the cost function can be expressed as

$$
\min J_{1}=\mathbf{W}_{1}\left\|\mathbf{M}_{c}-\mathbf{D}(\cdot) \boldsymbol{\delta}_{c}\right\|+\mathbf{W}_{2} \boldsymbol{\delta}_{\mathrm{RCS}},
$$

where $\mathbf{W}_{1} \in \Re^{3}, \mathbf{W}_{2} \in \Re^{n_{2}}$ are the weights to be designed.

Therefore, the control allocation problem is transformed into optimization problem to minimize the cost function (60) subject to (59).
In practical, on-off RCS jets can only provide the maximum torque or zero torque. Thus, $\delta_{\mathrm{RCS}}$ should be defined as binary variables. This paper employs the PWPF modulator to convert the continuous signal into on-off RCS commands. As shown in Figure 2, PWPF modulator consists of a low pass filer and a Schmitt trigger inside a feedback loop. $K_{m}$ and $T_{m}$ are the low pass filer gain. $u_{\text {on }}$ and $u_{\text {off }}$ are the on-value and off-value of Schmitt trigger.

\section{Numerical Simulation Results and Assessment}

In order to verify the effectiveness of proposed control methods, the comparisons between proposed control methods and two conventional methods are presented. The two conventional methods are FBL and boundary layer method based time-varying sliding mode control (BTVSMC).

The control algorithm of FBL can be expressed as [40]

$$
\mathbf{v}=\ddot{\mathbf{y}}_{\mathrm{c}}-k_{p} \mathbf{e}-k_{d} \dot{\mathbf{e}},
$$

where the parameters $k_{p}$ and $k_{d}$ should be a positive value.

As stated earlier, bound layer method is a conventional method to alleviate chattering phenomenon of SMC. Since DO is not employed in the control method, the control algorithm of BTVSMC is given by [17]

$$
\mathbf{v}=\mathbf{b}-\boldsymbol{\eta} \operatorname{sat}(\mathbf{s}) .
$$

The numerical tests in this paper employ a reentry vehicle, whose moments of inertia are $I_{x}=588791 \mathrm{~kg} \cdot \mathrm{m}^{2}, I_{y}=$ $1303212 \mathrm{~kg} \cdot \mathrm{m}^{2}$, and $I_{z}=1534164 \mathrm{~kg} \cdot \mathrm{m}^{2}$, and the products of inertia are $I_{z x}=I_{x z}=24242 \mathrm{~kg} \cdot \mathrm{m}^{2}$. In addition, the vehicle has a lifting-body configuration with 8 aerodynamic surfaces and 10 RCS jets. The aerodynamic surfaces include left outer elevon $\delta_{\text {elo }}$, right outer elevon $\delta_{\text {ero }}$, left inner elevon $\delta_{\text {eli }}$, right inner elevon $\delta_{\text {eri }}$, left flap $\delta_{\text {ff }}$, and right flap $\delta_{\text {fr }}$, left rudder $\delta_{\mathrm{rl}}$, and right rudder $\delta_{\mathrm{rr}}$ [47]. The constraints of the vector $\boldsymbol{\delta}_{\mathrm{A}}=\left[\delta_{\text {elo }}, \delta_{\text {ero }}, \delta_{\text {eli }}, \delta_{\text {eti }}, \delta_{\mathrm{fl}}, \delta_{\mathrm{fr}}, \delta_{\mathrm{rl}}, \delta_{\mathrm{rr}}\right]^{T}$ are given by

$$
\begin{aligned}
\boldsymbol{\delta}_{A \max } & =[0,0,0,0,30,30,30,30]^{T}, \\
\boldsymbol{\delta}_{A \min } & =-[25,25,25,25,10,10,30,30]^{T}, \\
\boldsymbol{\delta}_{d-A \max } & =-\boldsymbol{\delta}_{d-A i \min }=[10,10,10,10,10,10,10,10]^{T},
\end{aligned}
$$

where $\boldsymbol{\delta}_{A \max }$ and $\boldsymbol{\delta}_{A \text { min }}$ are measured in degree and $\boldsymbol{\delta}_{d-A \max }$ and $\boldsymbol{\delta}_{d-A i \min }$ are measured in degree per second.

Each RCS jet of reentry vehicle can produce $3559 \mathrm{~N}$ of thrust. In addition, the RCS torque matrix $\mathbf{D}_{\mathrm{RCS}}(\cdot)$ is defined as [48]

$$
\mathbf{D}_{\mathrm{RCS}}(\cdot)=\left[\begin{array}{cccccccccc}
0 & -2048 & 11625 & -6912 & 0 & 2054 & -11623 & 6912 & 0 & 0 \\
-498 & 0 & -9466 & 10944 & -498 & 0 & -9465 & 11798 & -498 & -498 \\
19897 & -15723 & -9465 & -11798 & -19897 & 15723 & 9465 & 11798 & -19897 & 19897
\end{array}\right] \text {. }
$$


TABLE 1: Sliding mode control parameters.

\begin{tabular}{|c|c|c|c|}
\hline Parameter\controller & BTVSMC & BTVSMC/DO & SOTVSMC/DO \\
\hline Sliding surface parameters & $\begin{array}{l}\mathbf{c}=\operatorname{diag}(2,2,2) \\
\lambda=2\end{array}$ & $\begin{array}{l}\mathbf{c}=\operatorname{diag}(2,2,2) \\
\lambda=2\end{array}$ & $\begin{array}{l}\mathbf{c}=\operatorname{diag}(2,2,2) \\
\lambda=2\end{array}$ \\
\hline Control parameters & $\begin{array}{l}\boldsymbol{\eta}=\operatorname{diag}(0.1,0.1,0.1) \\
\mathbf{h}=\operatorname{diag}(0.015,0.015,0.015)\end{array}$ & $\begin{array}{l}\boldsymbol{\eta}=\operatorname{diag}(0.1,0.1,0.1) \\
\mathbf{h}=\operatorname{diag}(0.015,0.015,0.015)\end{array}$ & $\begin{array}{l}\mathbf{k}_{1}=\operatorname{diag}(0.1,0.1,0.1) \\
\mathbf{k}_{2}=\operatorname{diag}(0.2,0.2,0.2) \\
\mathbf{k}_{3}=\operatorname{diag}(0.1,0.1,0.1) \\
\mathbf{k}_{4}=\operatorname{diag}(0.1,0.1,0.1) \\
m=3\end{array}$ \\
\hline DO parameters & & $\begin{array}{l}\gamma_{1}=8.2, \gamma_{2}=4.1, \gamma_{3}=2.0 \\
\mathbf{L}=\operatorname{diag}(0.005,0.005,0.01)\end{array}$ & $\begin{array}{l}\gamma_{1}=8.2, \gamma_{2}=4.1, \gamma_{3}=2.0 \\
\mathbf{L}=\operatorname{diag}(0.005,0.005,0.01)\end{array}$ \\
\hline
\end{tabular}

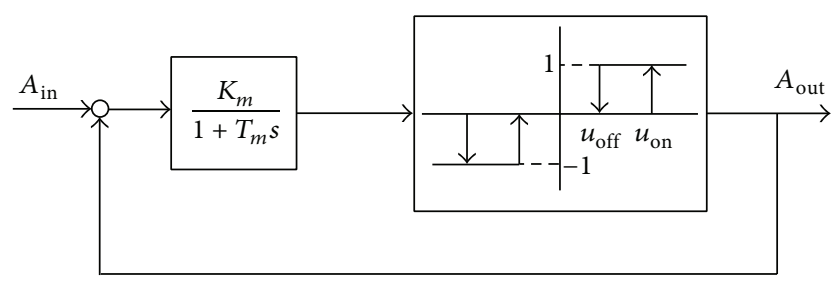

Figure 2: PWPF modulator.

The initial conditions for reentry vehicle are taken as follows: the altitude $h=55.0 \mathrm{~km}$, Mach number $\mathrm{Ma}=9.8$, $\Omega_{0}=\left[32.0^{\circ}, 2.0^{\circ}, 58.0^{\circ}\right]^{T}$, and $\mathbf{w}_{0}=\left[0.0^{\circ} / \mathrm{s}, 0.0^{\circ} / \mathrm{s}, 0.0^{\circ} / \mathrm{s}\right]^{T}$. And attitude angle commands are set to be $\Omega_{c}=\left[30.0^{\circ}, 0.0^{\circ}\right.$, $\left.60.0^{\circ}\right]^{T}$. The reentry vehicle suffers high structural stresses for high Mach number and bad aerodynamics for large AOA, and, hence, the rudders are not allowed to be used [10].

Furthermore, additional constraints for control allocation problem should be satisfied:

$$
\delta_{\mathrm{rl}}=\delta_{\mathrm{rr}}=0
$$

The planet uncertainties are set in consideration of 5 percent bias conditions for moments of inertia and products of inertia, 10 percent bias conditions for aerodynamic coefficients, and 10 percent bias conditions for atmospheric density. In addition, the external disturbance torque vector takes the form of

$$
\Delta \mathbf{M}=\left[\begin{array}{l}
0.5+\sin (0.1 t)+\sin (t) \\
0.5+\sin (0.1 t)+\sin (t) \\
0.5+\sin (0.1 t)+\sin (t)
\end{array}\right] \times 10^{4} \mathrm{~N} \cdot \mathrm{m}
$$

To validate the robustness and the chattering reduction of the proposed methods, numerical simulations of FBL (61), BTVSMC (62), BTVSMC/DO (36), and SOTVSMC/DO (45) are presented. The overall attitude control system architecture for reentry is showed in Figure 1. And the integration step is
TABLE 2: Control allocation parameters.

\begin{tabular}{lc}
\hline Parameter & Value \\
\hline $\mathbf{W}_{1}$ & {$[1,1,1]$} \\
$\mathbf{W}_{2}$ & {$[0.1, \ldots, 0.1]$} \\
$K_{m}$ & 4.5 \\
$T_{m}$ & 0.15 \\
$u_{\text {on }}$ & 0.45 \\
$u_{\text {off }}$ & 0.15 \\
\hline
\end{tabular}

specified as 0.01 seconds. In FBL (61), the control parameters are selected as $k_{p}=k_{d}=3$. In addition, the control parameters of the sliding mode controllers are specified in Table 1. After the command control torque vector, $\mathbf{M}_{c}$, is produced by these controllers, the actuator command is obtained by control allocation algorithm as presented in Section 5. The parameters of control allocation problem are as shown in Table 2.

The variations of the attitude angles including AOA, sideslip angle, and bank angle under FBL, BTVSMC, BTVS$\mathrm{MC} / \mathrm{DO}$, and SOTVSMC/DO are shown in Figure 3. It is obvious that SMC has significant robustness performance in the presence of uncertainties and disturbances. Since FBL relies on the knowledge of the exact model dynamics, the tracking errors under FBL do not converge to zero. The second row of Figure 3 shows the attitude angle evolutions in steady-state region. Compared with FBL and BTVSMC, BTVSMC/DO and SOTVSMC/DO achieve the goals of tracking with higher accuracy.

To further evaluate the tracking performance between BTVSMC/DO and SOTVSMC/DO, the local view of attitude angle evolutions via BTVSMC/DO and SOTVSMC/DO is shown in Figure 4 . The results suggest that all the attitude angles converge to the desired values within 4 seconds in the transient region for both methods. As shown in the local view of attitude angles in the steady-state region, the tracking errors under SOTVSMC/DO are much smaller than those under BTVSMC/DO. 

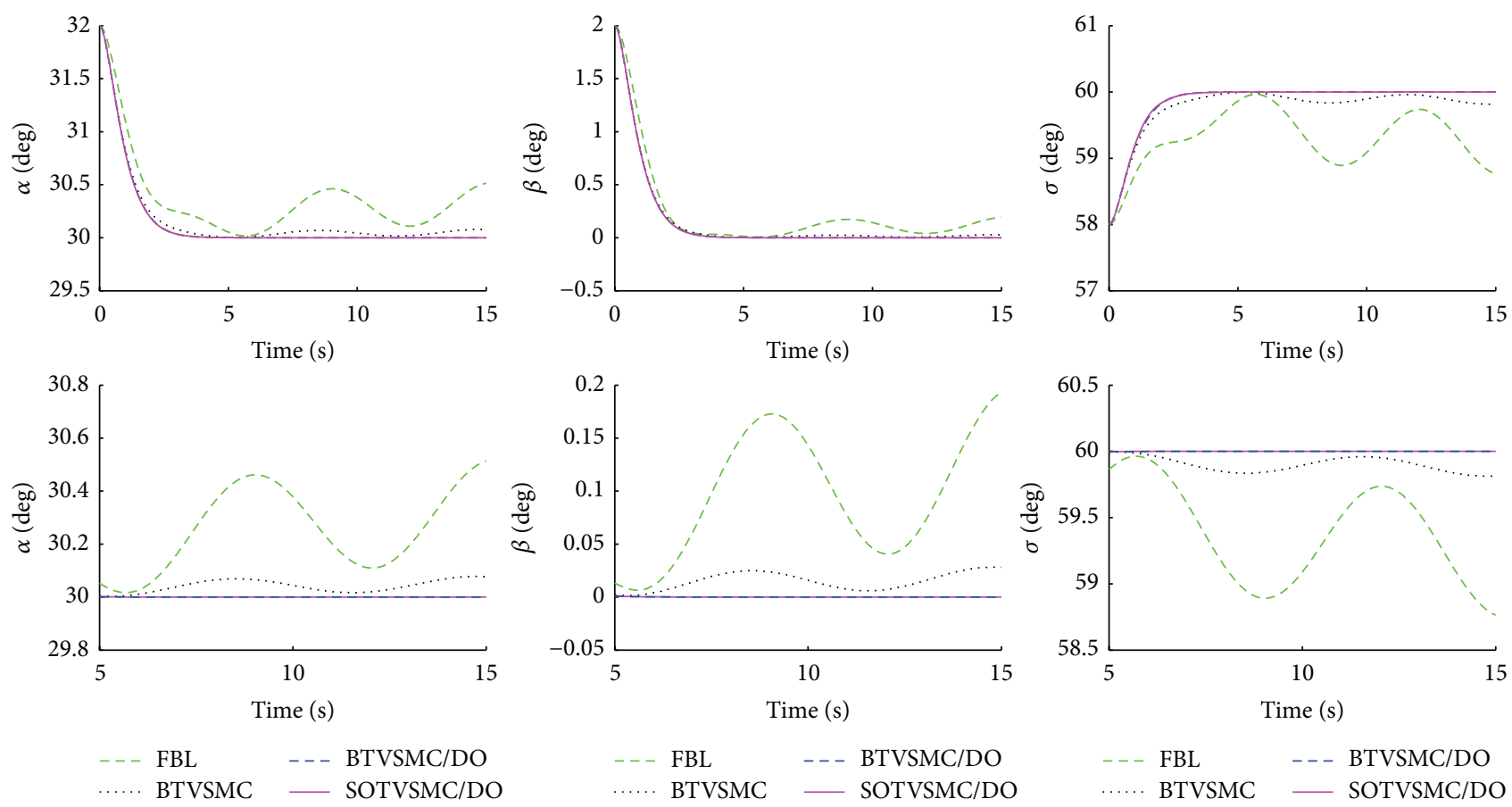

FIgURE 3: Comparison of attitude angle evolutions via FBL, BTVSMC, BTVSMC/DO, and SOTVSMC/DO.
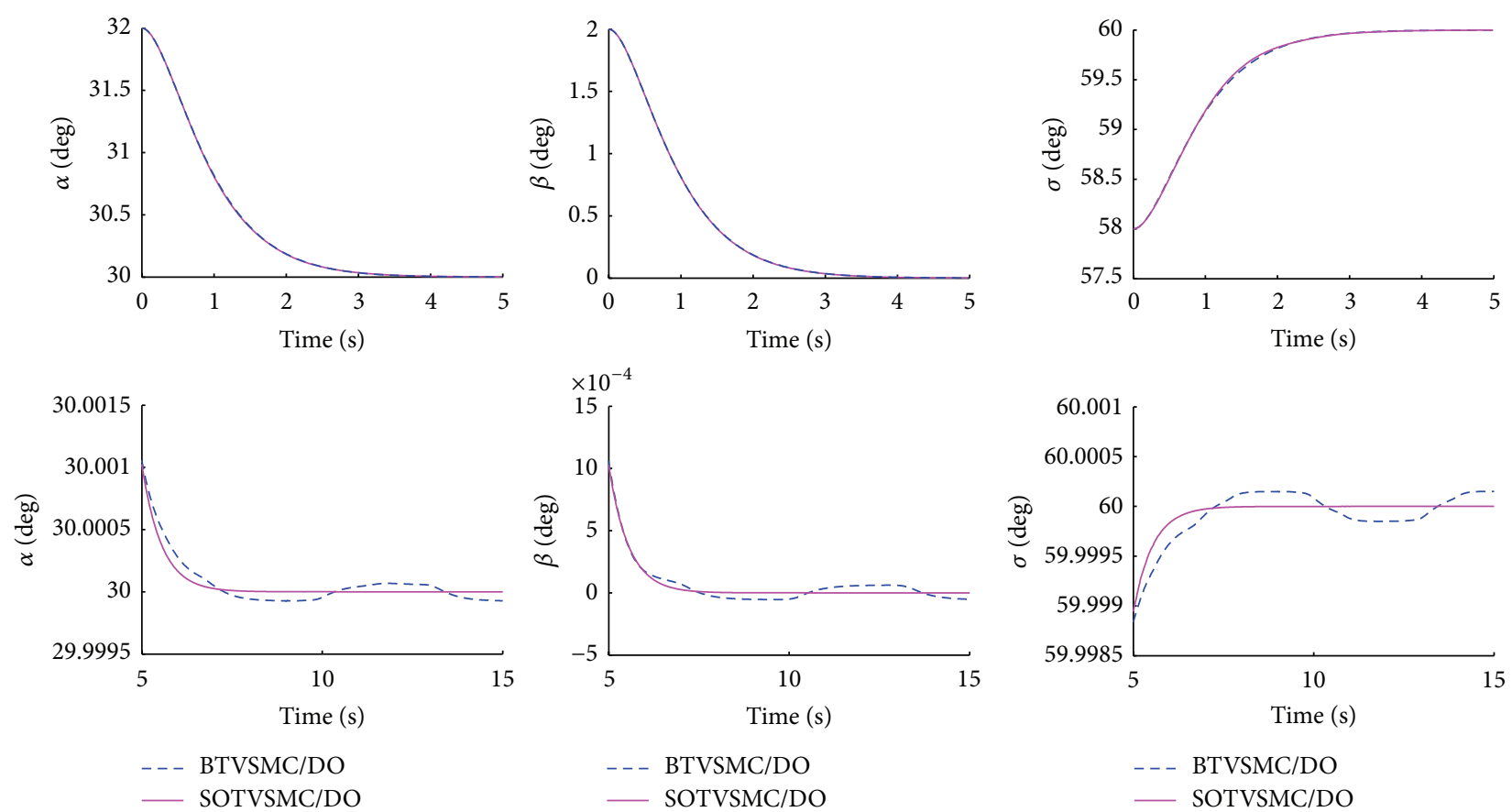

FIGURE 4: Local view of attitude angle evolutions via BTVSMC/DO and SOTVSMC/DO.

The sliding surface responses via BTVSMC, BTVSMC/ DO, and SOTVSMC/DO are shown in Figure 5. It is obvious that the sliding surface under BTVSMC does not converge to zero because DO is not introduced. The system trajectories under BTVSMC/DO and SOTVSMC/DO move apart from zero after the start point, and move back to zero around 4 seconds later. The second row of Figure 5 shows the local view of steady-state region under BTVSMC/DO and 

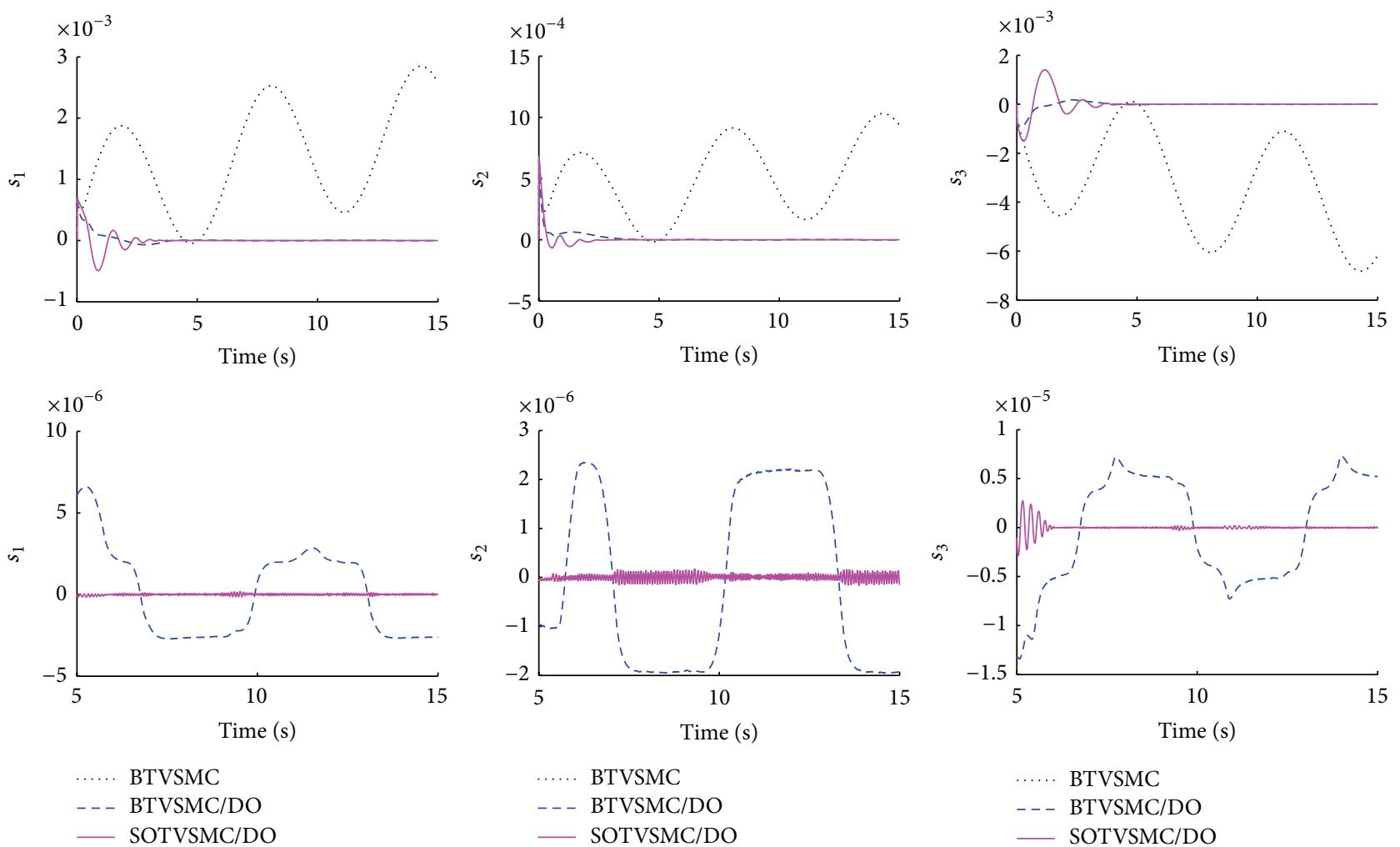

FIGURE 5: Comparison of sliding surface responses via BTVSMC, BTVSMC/DO, and SOTVSMC/DO.

SOTVSMC/DO. As boundary layer method is applied, the sliding surface stays inside of the boundary layer; however, the tracking accuracy within the boundary layer is not guaranteed. As a result, the sliding surface under SOTVSMC/ DO converges to zero more precisely.

The estimations of the sliding surface by BTVSMC/DO and SOTVSMC/DO are shown in Figures 6(a) and 6(b) respectively. In addition, Figure 7 depicts the estimations of uncertainty terms. Clearly, all of sliding surfaces and lumped uncertainty can be estimated by DO effectively.

Figure 8 illustrates the comparison of attitude angular rate evolutions via FBL, BTVSMC, BTVSMC/DO, and SOTVSMC/DO. And the produced command control torques are showed in Figure 9. Control chattering is undesirable in practice because it involves high control activity and may excite high frequency unmolded dynamics. As is shown in Figure 9, the problem of chattering phenomenon for SMC is eliminated in the cases of BTVSMC, BTVSMC/DO, and SOTVSMC/DO.

This paper formulates all the numerical simulations in consideration of the constraints for aerodynamic deflections and RCS thrusts, both of which are actuator command and can be obtained by control allocation algorithm presented in Section 5. The aerodynamic deflections via BTVSMC/DO and SOTVSMC/DO are shown in Figures 10(a) and 11(a), respectively. The rudders are kept around zero. All the actuator limits in (59) and (65) are satisfied. The RCS thrust commands via BTVSMC/DO and SOTVSMC/DO are shown in Figures 10(b) and 11(b), respectively. The RCS thrusts compensate the torque errors caused by aerodynamic deflection saturation with high accuracy.

\section{Conclusion}

This paper investigates the finite-time control problem of reentry vehicle with aerodynamic surfaces and RCS jets and seeks for more reliable attitude controller design and the control allocation design. The numerical simulation results prove the tracking accuracy and robustness of the proposed attitude controller and control allocation method.

For attitude controller design, two kinds of robust finitetime TVSMC controllers are proposed. Time-varying sliding surface is employed to eliminate the reaching phase of transit dynamics, and thus the global robustness is guaranteed. A DO is introduced to enhance the robustness against planet uncertainness and external disturbances. To alleviate the chattering, boundary layer method and second-order SMC method are employed, respectively. Both of the proposed methods can avoid chattering phenomenon effectively. Moreover, the tracking error under SOTVSMC/DO converges to zero more precisely.

A control allocation approach is introduced to generate the actuator commands, including aerodynamic surface deflections and on-off RCS thrusts. The nondeterministic 

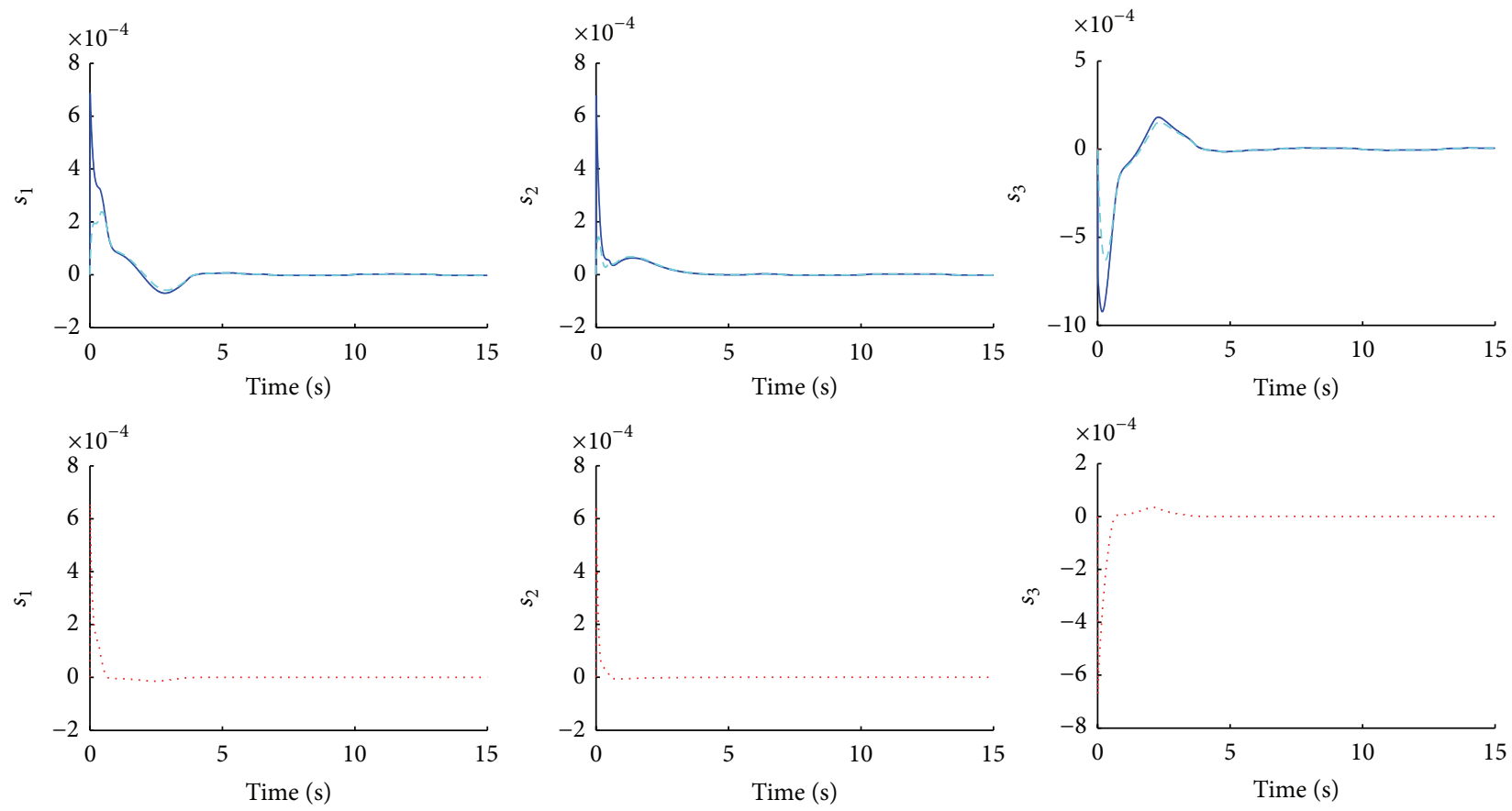

- Real
-_- Estimated

Estimation error

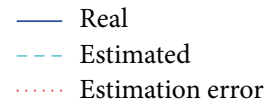

- Real

- - Estimated

Estimation error

(a) Comparison between $\mathbf{s}$ and $\mathbf{z}_{0}$ under BTVSMC/DO
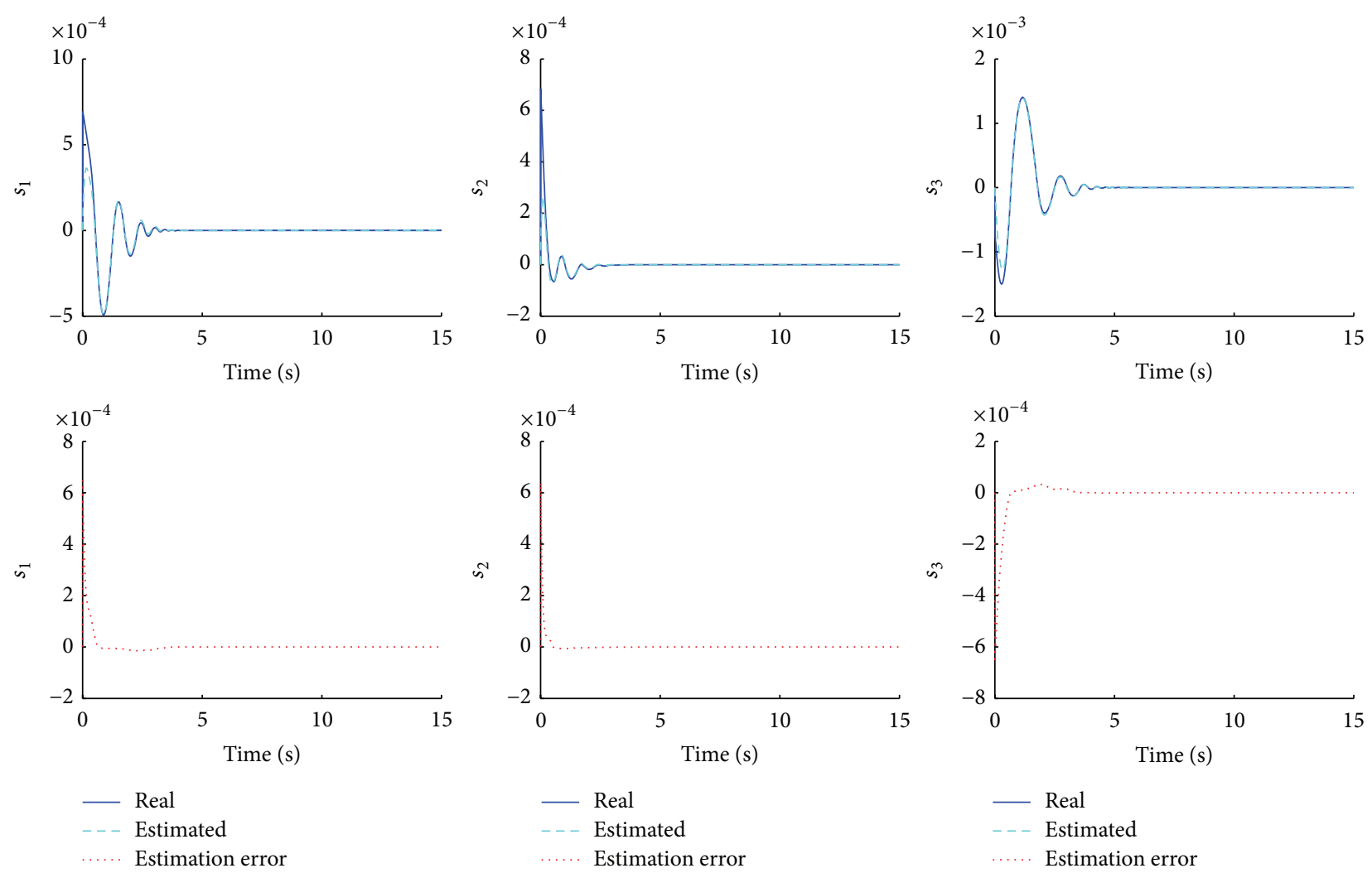

(b) Comparison between $\boldsymbol{s}$ and $\mathbf{z}_{0}$ under SOTVSMC/DO

FIgURE 6: The estimations of sliding surface by BTVSMC/DO and SOTVSMC/DO. 

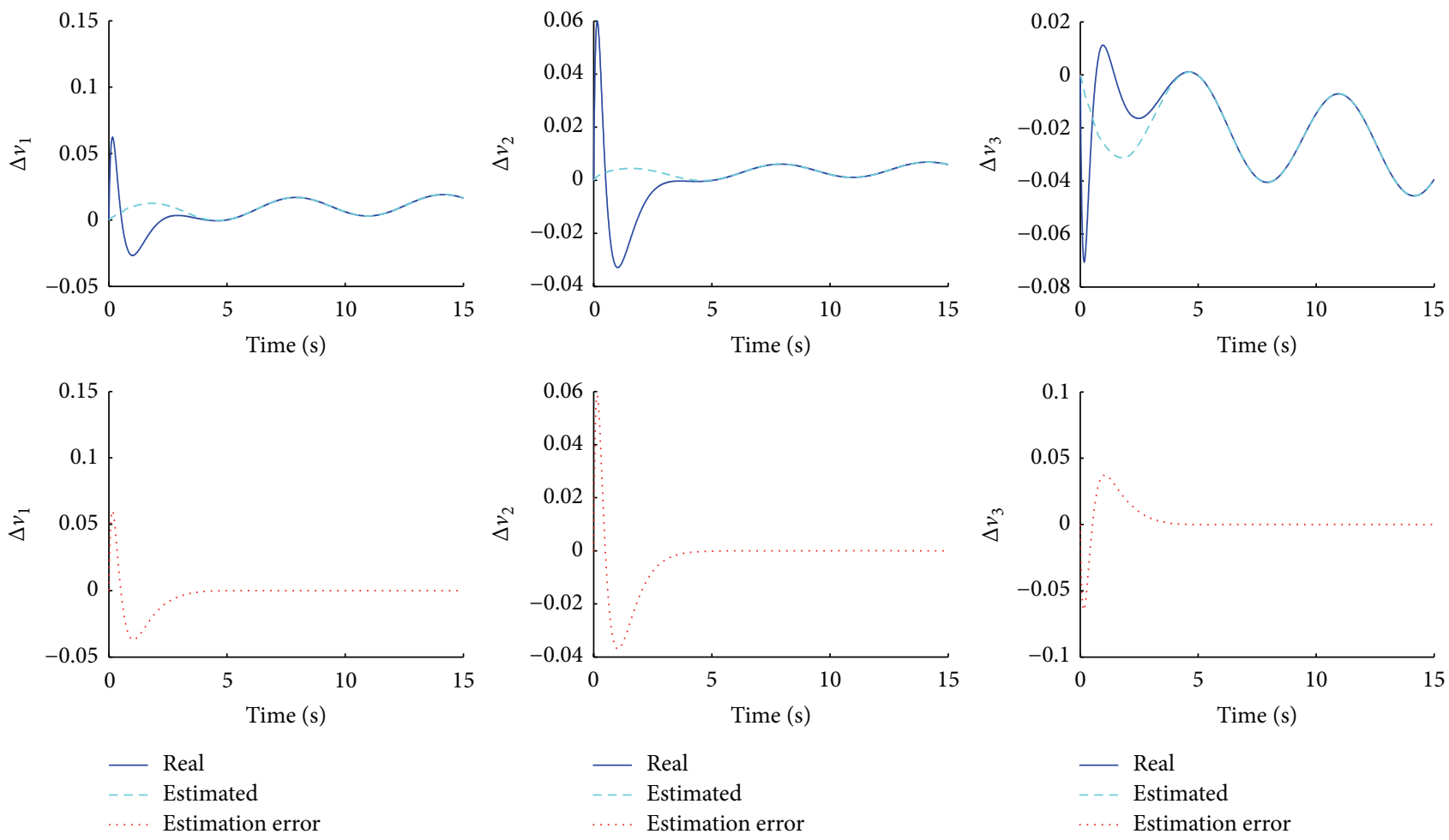

(a) Comparison between $\Delta \mathbf{v}$ and $\mathbf{z}_{1}$ under BTVSMC/DO
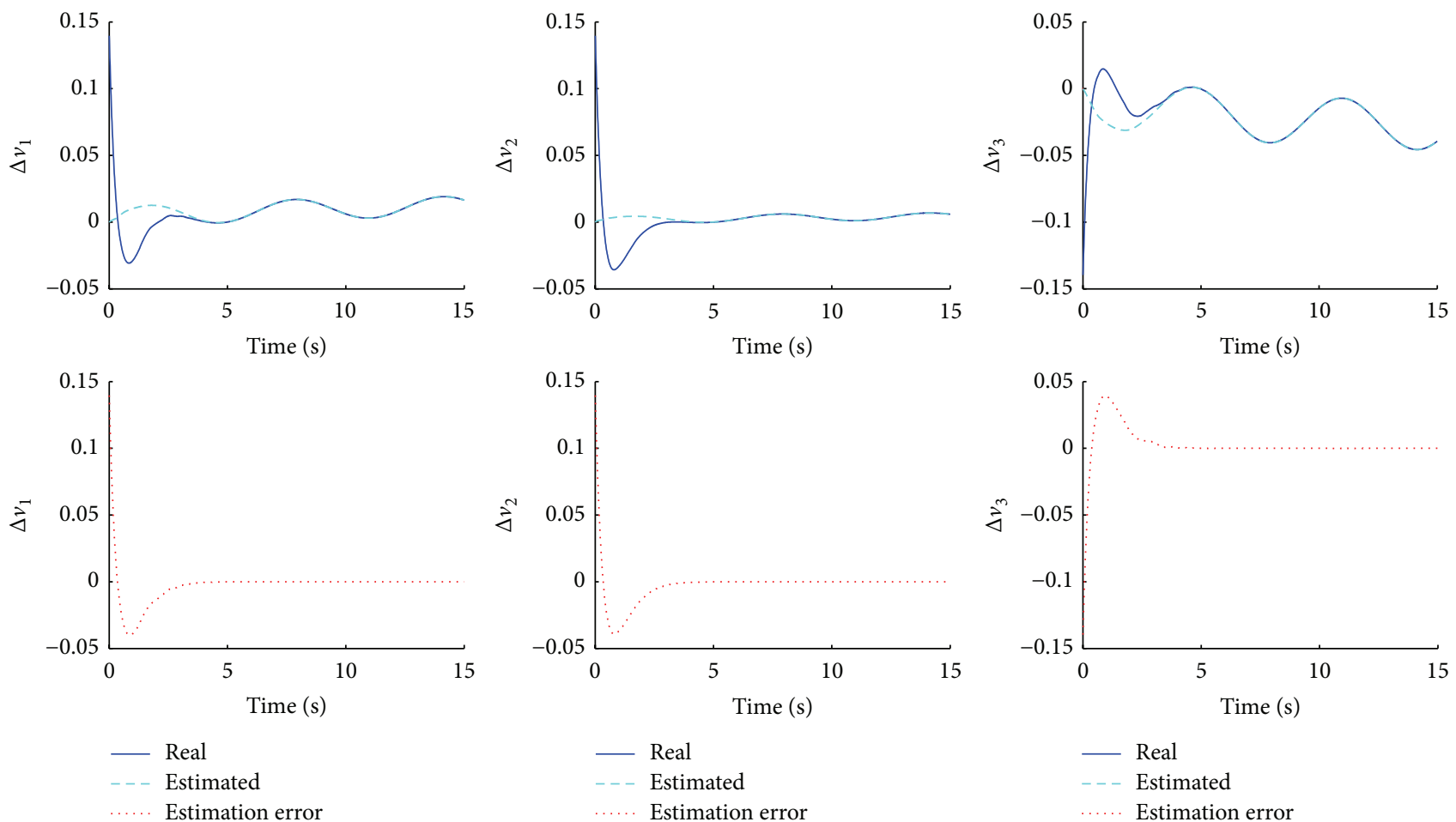

(b) Comparison between $\Delta \mathbf{v}$ and $\mathbf{z}_{1}$ under SOTVSMC/DO

FIGURE 7: The estimations of the uncertainty terms by BTVSMC/DO and SOTVSMC/DO. 

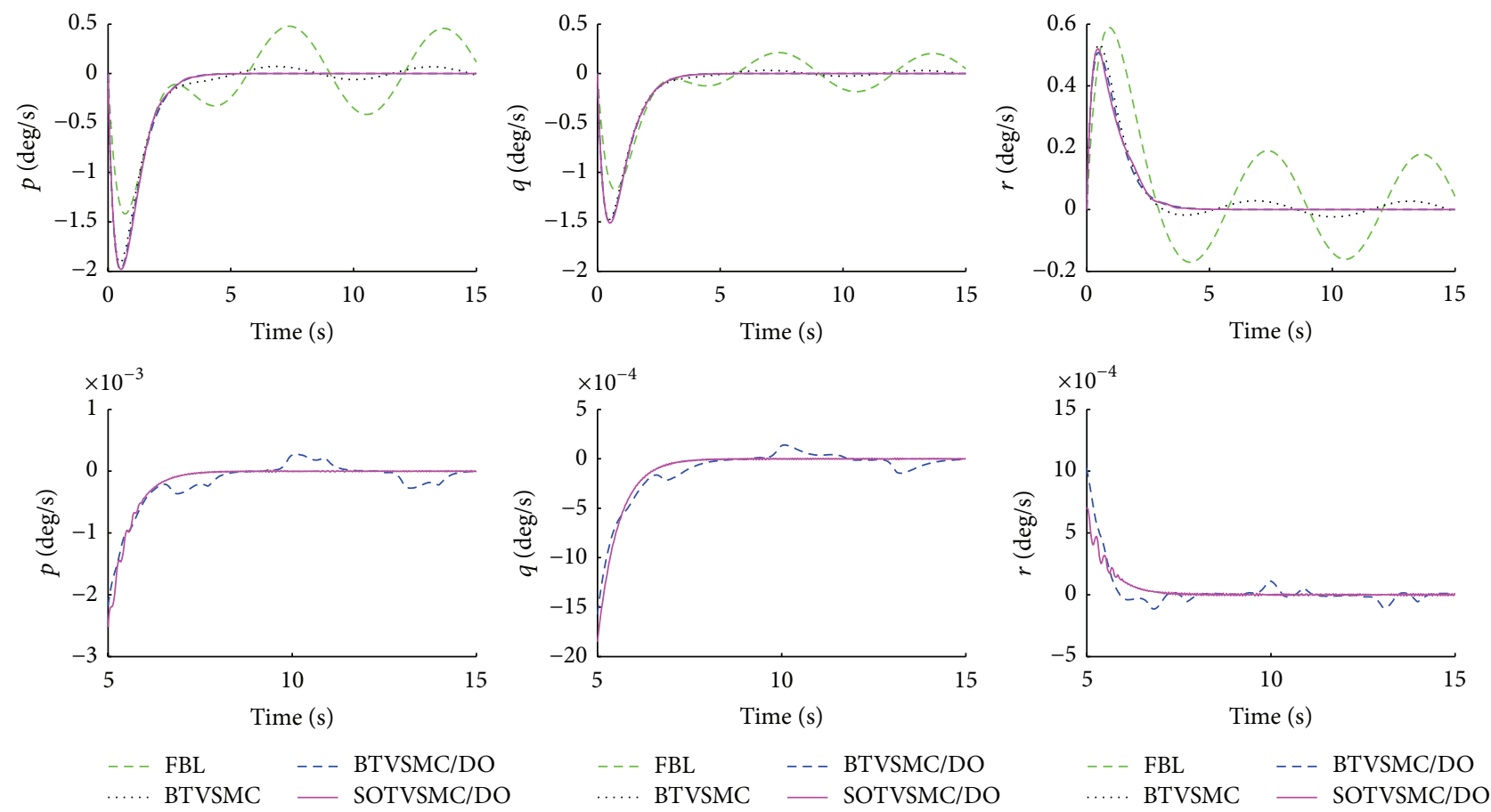

Figure 8: Comparison of attitude angular rate evolutions via FBL, BTVSMC, BTVSMC/DO, and SOTVSMC/DO.
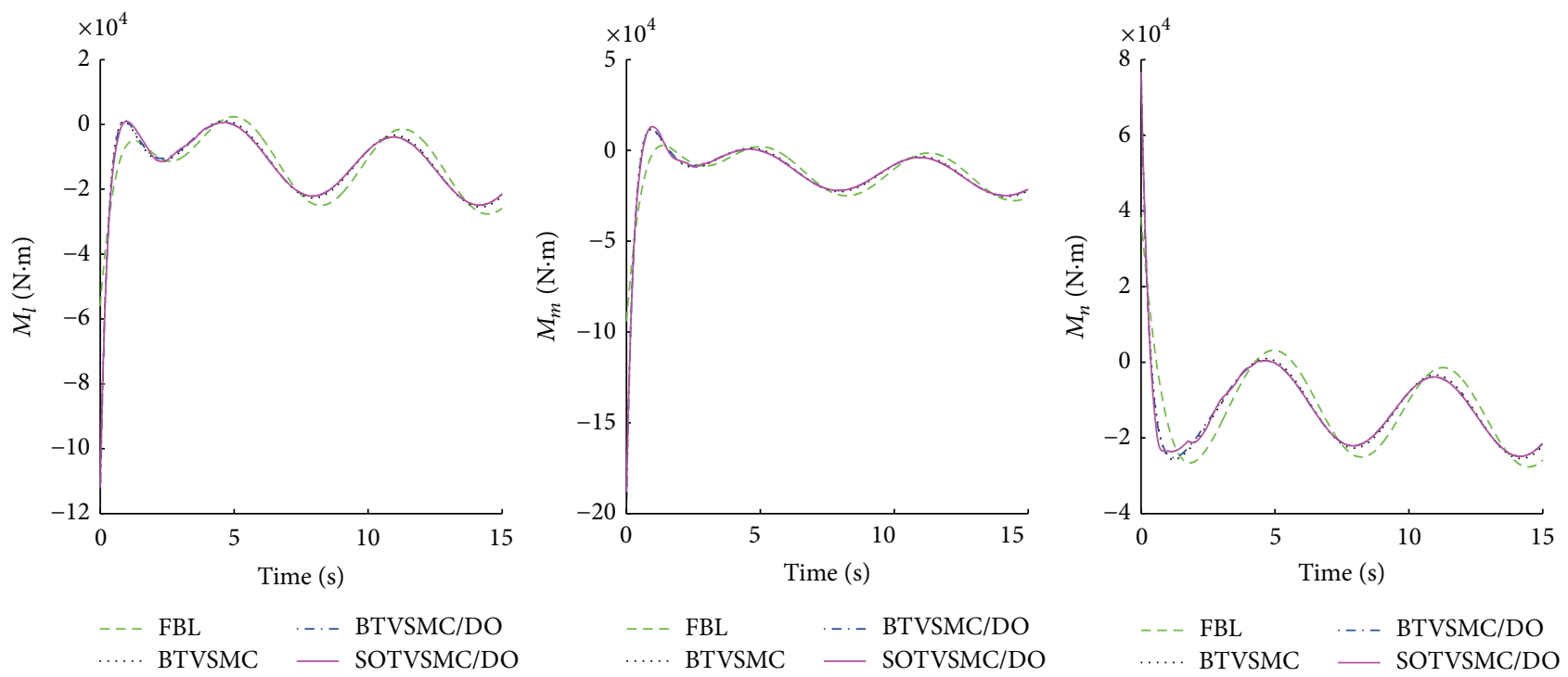

FIgURE 9: Comparison of command control torque via FBL, BTVSMC, BTVSMC/DO, and SOTVSMC/DO.

system equations are solved by optimization methods, and the proposed weighted cost function is capable of minimizing both the utilization of RCS jets and the differences between the desired torque and the torque produced by actuators.

\section{Conflict of Interests}

The authors declare that there is no conflict of interests regarding the publication of this paper. 

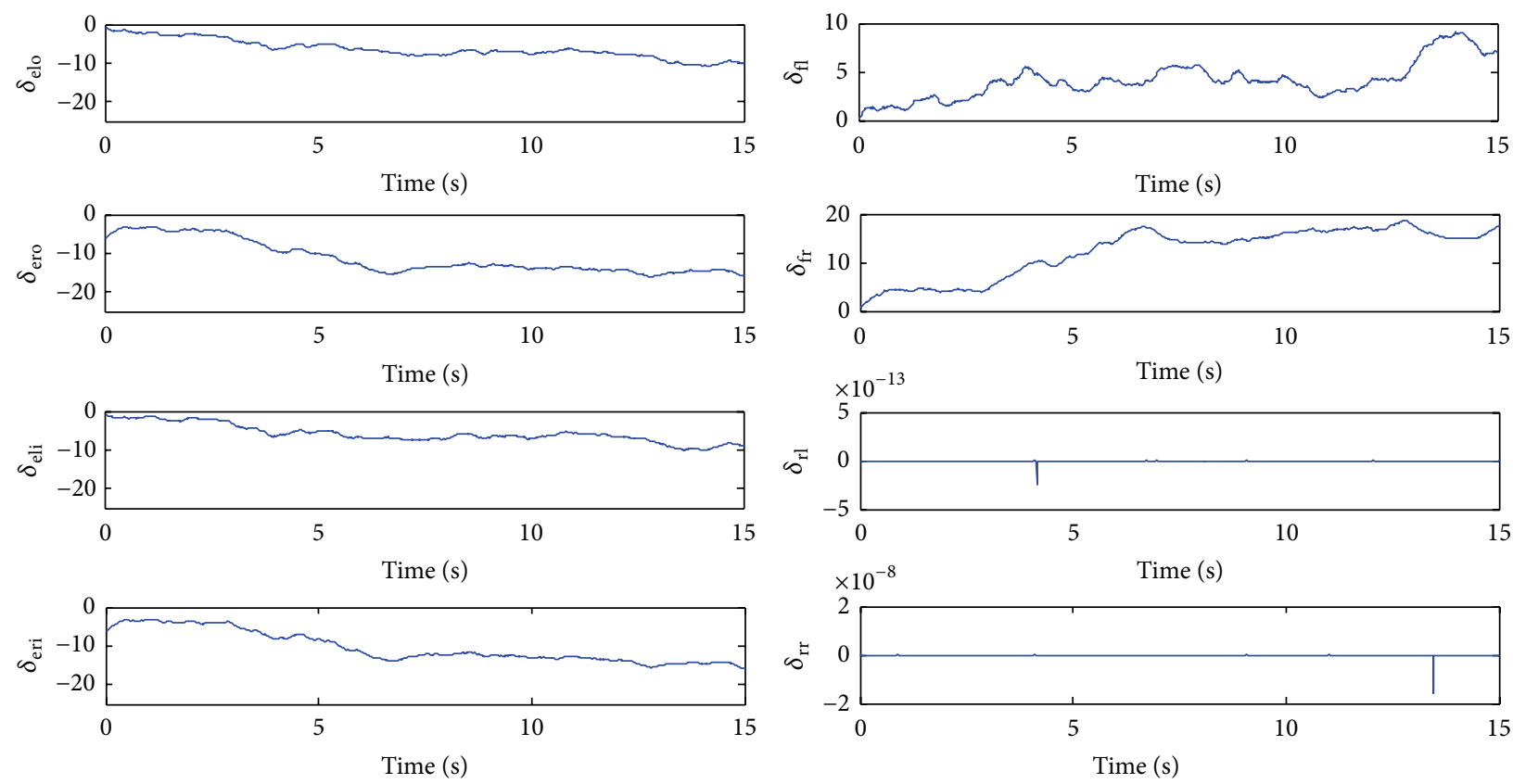

(a) Elevon, flap, and rudder commands
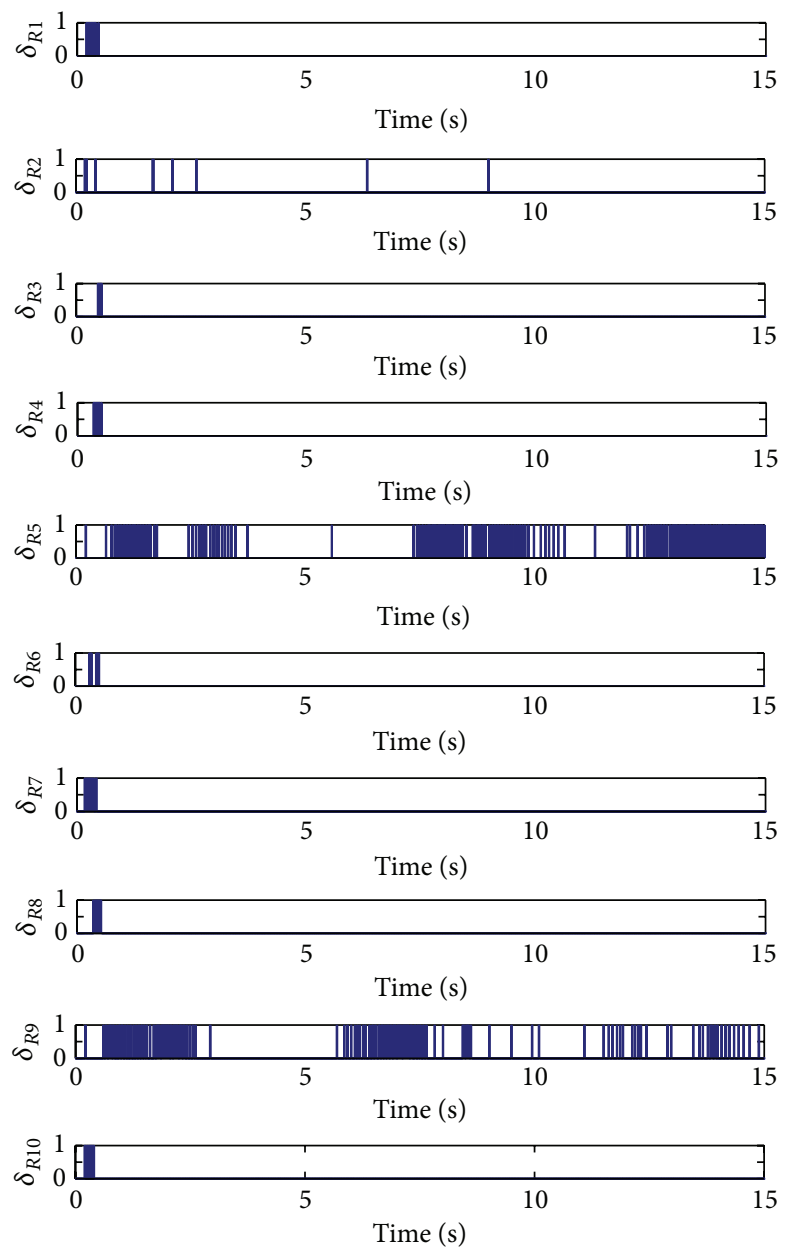

(b) RCS commands

FIGURE 10: Aerodynamic surface commands and RCS commands via BTVSMC/DO. 

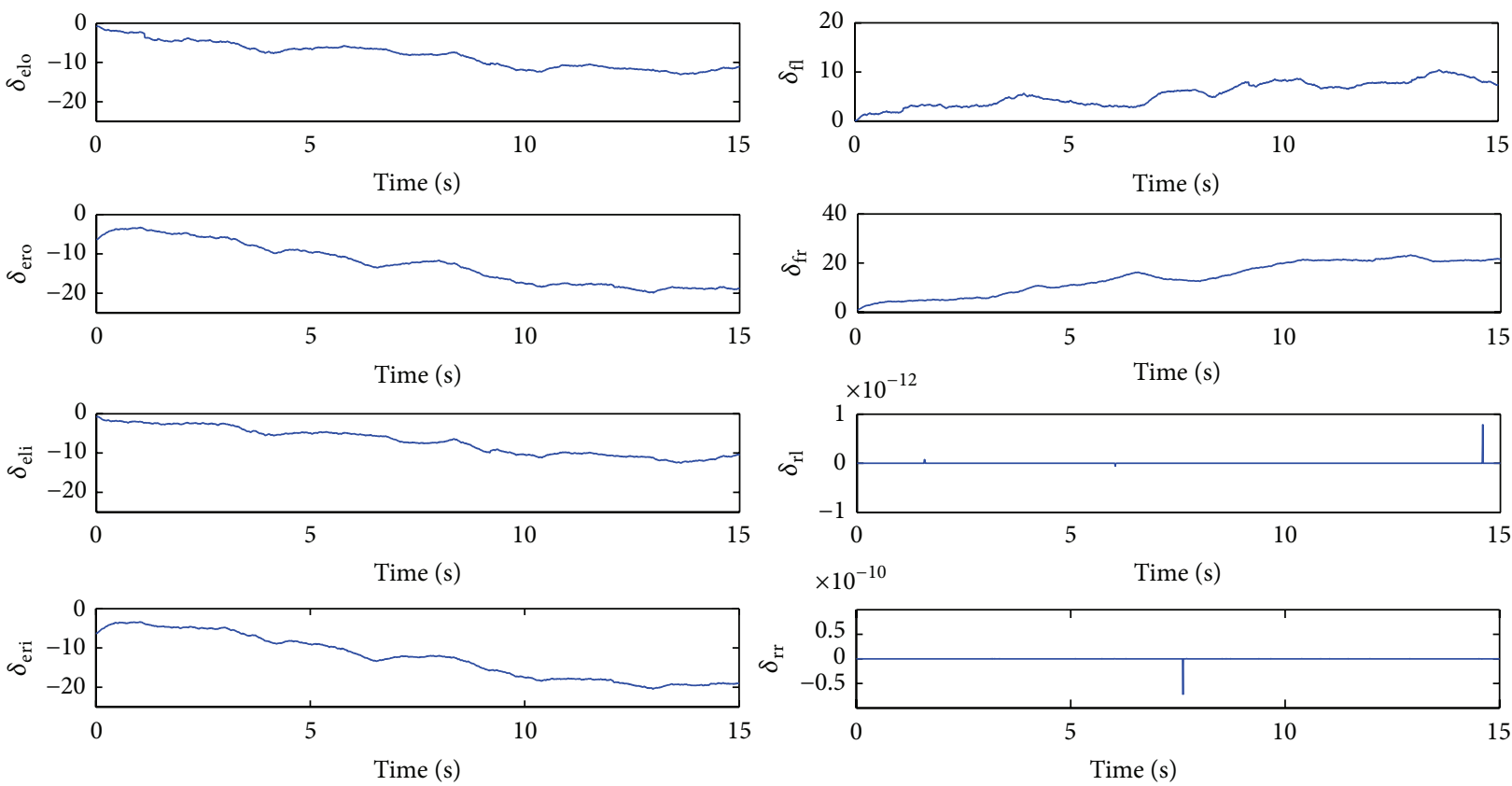

(a) Elevon, flap, and rudder commands
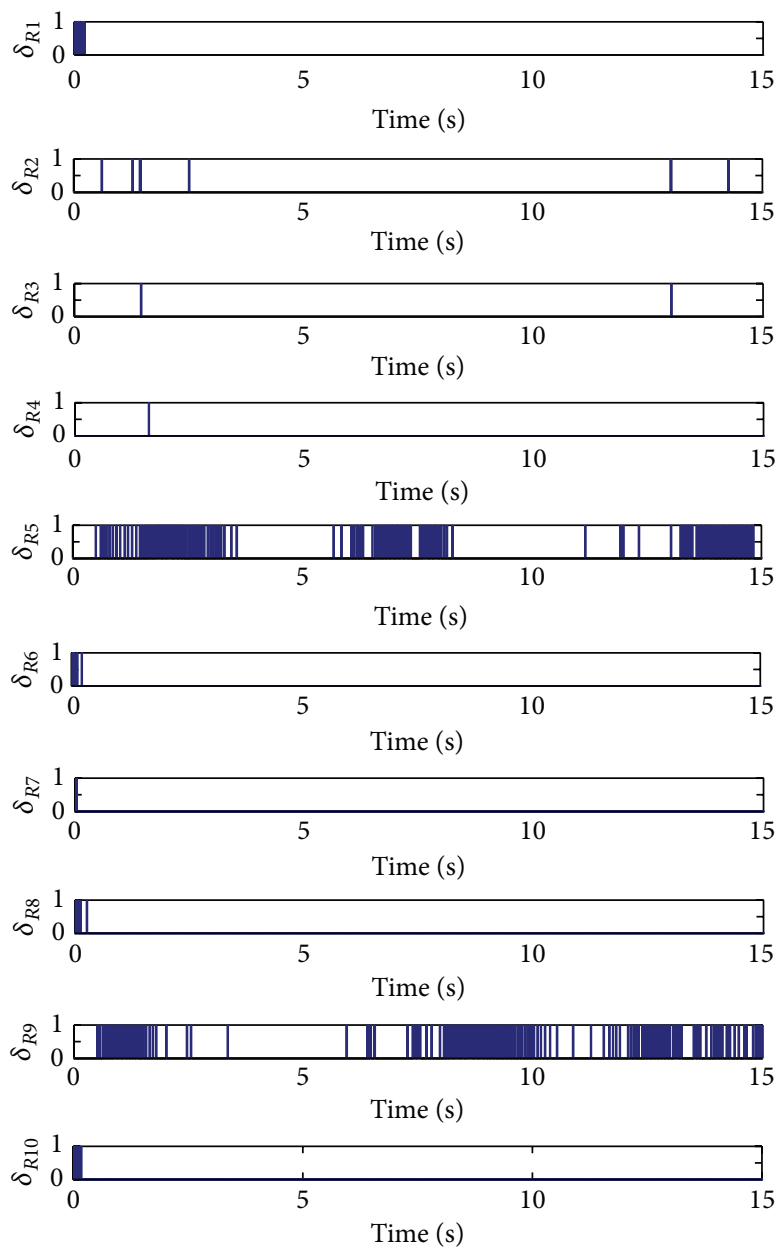

(b) RCS commands

FIGURE 11: Aerodynamic surface commands and RCS commands via SOTVSMC/DO. 


\section{Acknowledgments}

The authors would like to appreciate the editor and all the anonymous reviewers for their comments, which helped to improve the quality of this paper. This study was supported by National Natural Science Foundation of China (11202024).

\section{References}

[1] Z. Shen and P. Lu, "Onboard generation of three-dimensional constrained entry trajectories," Journal of Guidance, Control, and Dynamics, vol. 26, no. 1, pp. 111-121, 2003.

[2] R. Smith and A. Ahmed, "Robust parametrically varying attitude controller designs for the X-33 vehicle," in AIAA Guidance, Navigation, and Control Conference and Exhibit, Denver, Colo, USA, 2000, AIAA-2000-4158.

[3] W. J. Rugh and J. S. Shamma, "Research on gain scheduling," Automatica, vol. 36, no. 10, pp. 1401-1425, 2000.

[4] D. J. Leith and W. E. Leithead, "Survey of gain-scheduling analysis and design," International Journal of Control, vol. 73, no. 11, pp. 1001-1025, 2000.

[5] R. M. Agustin, R. S. Mangoubi, R. M. Hain, and N. J. Adams, "Robust failure detection for reentry vehicle attitude control systems," Journal of Guidance, Control, and Dynamics, vol. 22, no. 6, pp. 839-845, 1999.

[6] P. Lu, "Regulation about time-varying trajectories: precision entry guidance illustrated," Journal of Guidance, Control, and Dynamics, vol. 22, no. 6, pp. 784-790, 1999.

[7] A. Rahideh, A. H. Bajodah, and M. H. Shaheed, "Real time adaptive nonlinear model inversion control of a twin rotor MIMO system using neural networks," Engineering Applications of Artificial Intelligence, vol. 25, no. 6, pp. 1289-1297, 2012.

[8] W. R. van Soest, Q. P. Chu, and J. A. Mulder, "Combined feedback linearization and constrained model predictive control for entry flight," Journal of Guidance, Control, and Dynamics, vol. 29, no. 2, pp. 427-434, 2006.

[9] H. Xu, M. D. Mirmirani, and P. A. Ioannou, "Adaptive sliding mode control design for a hypersonic flight vehicle," Journal of Guidance, Control, and Dynamics, vol. 27, no. 5, pp. 829-838, 2004.

[10] R. R. Da Costa, Q. P. Chu, and J. A. Mulder, "Reentry flight controller design using nonlinear dynamic inversion," Journal of Spacecraft and Rockets, vol. 40, no. 1, pp. 64-71, 2003.

[11] K. D. Young, V. I. Utkin, and Ü. Özgüner, "A control engineer's guide to sliding mode control," IEEE Transactions on Control Systems Technology, vol. 7, no. 3, pp. 328-342, 1999.

[12] A. Pisano and E. Usai, "Sliding mode control: a survey with applications in math," Mathematics and Computers in Simulation, vol. 81, no. 5, pp. 954-979, 2011.

[13] J. Y. Hung, W. Gao, and J. C. Hung, "Variable structure control: a survey," IEEE Transactions on Industrial Electronics, vol. 40, no. 1, pp. 2-22, 1993.

[14] O. Barambones Caramazana, P. Alkorta Egiguren, and J. M. González de Durana García, "Sliding mode position control for real-time control of induction motors," International Journal of Innovative Computing, Information and Control, vol. 9, pp. 27412754, 2013.

[15] L. Wu, X. Su, and P. Shi, "Sliding mode control with bounded $L_{2}$ gain performance of Markovian jump singular time-delay systems," Automatica, vol. 48, no. 8, pp. 1929-1933, 2012.
[16] Y. Shtessel, C. Tournes, and D. Krupp, "Reusable launch vehicle control in sliding modes," in Proceedings of the Guidance, Navigation, and Control Conference, AIAA-1997-3533, American Institute of Aeronautics and Astronautics, 1997.

[17] Y. Shtessel, J. McDuffie, M. Jackson et al., "Sliding mode control of the X-33 vehicle in launch and re-entry modes in," in Proceedings of the Guidance, Navigation, and Control Conference and Exhibit, AIAA-1998-4414, American Institute of Aeronautics and Astronautics, 1998.

[18] Y. Shtessel, C. Hall, and M. Jackson, "Reusable launch vehicle control in multiple-time-scale sliding modes," Journal of Guidance, Control, and Dynamics, vol. 23, no. 6, pp. 1013-1020, 2000.

[19] Y. Shtessel, J. Zhu, and D. Dan, "Reusable launch vehicle attitude control using time-varying sliding modes," in Proceedings of the AIAA Guidance, Navigation, and Control Conference and Exhibit, AIAA-2002-4779, American Institute of Aeronautics and Astronautics, 2002.

[20] M. Defoort, T. Floquet, A. Kokosy, and W. Perruquetti, "A novel higher order sliding mode control scheme," Systems \& Control Letters, vol. 58, no. 2, pp. 102-108, 2009.

[21] L. Wu, W. X. Zheng, and H. Gao, "Dissipativity-based sliding mode control of switched stochastic systems," IEEE Transactions on Automatic Control, vol. 58, no. 3, pp. 785-791, 2013.

[22] H. Sun, S. Li, and C. Sun, "Finite time integral sliding mode control of hypersonic vehicles," Nonlinear Dynamics, vol. 73, no. 1-2, pp. 229-244, 2013.

[23] M. R. Soltanpour, B. Zolfaghari, M. Soltani, and M. H. Khooban, "Fuzzy sliding mode control design for a class of nonlinear systems with structured and unstructured uncertainties," International Journal of Innovative Computing, Information and Control, vol. 9, no. 7, pp. 2713-2726, 2013.

[24] X. Su, P. Shi, L. Wu, and M. V. Basin, "Reliable filtering with strict dissipativity for T-S fuzzy time-delay systems," IEEE Transactions on Cybernetics, 2014.

[25] H. Lee and V. I. Utkin, "Chattering suppression methods in sliding mode control systems," Annual Reviews in Control, vol. 31, no. 2, pp. 179-188, 2007.

[26] H. Lee, E. Kim, H.-J. Kang, and M. Park, "A new sliding-mode control with fuzzy boundary layer," Fuzzy Sets and Systems, vol. 120, no. 1, pp. 135-143, 2001.

[27] A. Levant, Higher order sliding modes and their application for controlling uncertain processes [Ph.D. thesis], Institute for System Studies of the USSR Academy of Science, Moscow, Russia, 1987.

[28] A. Levant, "Sliding order and sliding accuracy in sliding mode control," International Journal of Control, vol. 58, no. 6, pp. 12471263, 1993.

[29] A. Levant, "Robust exact differentiation via sliding mode technique," Automatica, vol. 34, no. 3, pp. 379-384, 1998.

[30] G. Bartolini, A. Ferrara, and E. Usai, "Output tracking control of uncertain nonlinear second-order systems," Automatica, vol. 33, no. 12, pp. 2203-2212, 1997.

[31] G. Bartolini, A. Ferrara, and E. Usai, "Chattering avoidance by second-order sliding mode control," IEEE Transactions on Automatic Control, vol. 43, no. 2, pp. 241-246, 1998.

[32] K. Ohishi, M. Nakao, K. Ohnishi, and K. Miyachi, "Microprocessor-controlled DC motor for load-insensitive position servo system," IEEE Transactions on Industrial Electronics, vol. IE-34, no. 1, pp. 44-49, 1985.

[33] C. E. Hall and Y. B. Shtessel, "Sliding mode disturbance observer-based control for a reusable launch vehicle," Journal of Guidance, Control, and Dynamics, vol. 29, no. 6, pp. 1315-1328, 2006. 
[34] Y. B. Shtessel, I. A. Shkolnikov, and A. Levant, "Smooth secondorder sliding modes: missile guidance application," Automatica, vol. 43, no. 8, pp. 1470-1476, 2007.

[35] S. N. D’Souza and N. Sarigul-Klijn, "Survey of planetary entry guidance algorithms," Progress in Aerospace Sciences, vol. 68, pp. 64-74, 2014.

[36] P. Lu, "Entry guidance: a unified method," Journal of Guidance, Control, and Dynamics, vol. 37, no. 3, pp. 713-728, 2014.

[37] B. Tian, Q. Zong, J. Wang, and F. Wang, "Quasi-continuous high-order sliding mode controller design for reusable launch vehicles in reentry phase," Aerospace Science and Technology, vol. 28, no. 1, pp. 198-207, 2013.

[38] N. X. Vinh, A. Busemann, and R. D. Culp, Hypersonic and Planetrary Entry Flight Mechanics, The University of Michigan Press, 1980.

[39] T. A. Johansen and T. I. Fossen, "Control allocation-a survey," Automatica, vol. 49, no. 5, pp. 1087-1103, 2013.

[40] A. J. Krener, "Feedback linearization," in Mathematical Control Theory, J. Baillieul and J. C. Willems, Eds., pp. 66-98, Springer, New York, NY, USA, 1999.

[41] C. Binglong, L. Xiangdong, and C. Zhen, "Exponential timevarying sliding mode control for large angle attitude eigenaxis maneuver of rigid spacecraft," Chinese Journal of Aeronautics, vol. 23, no. 4, pp. 447-453, 2010.

[42] A. Levant, "Higher-order sliding modes, differentiation and output-feedback control," International Journal of Control, vol. 76, no. 9-10, pp. 924-941, 2003.

[43] S. P. Bhat and D. S. Bernstein, "Finite-time stability of continuous autonomous systems," SIAM Journal on Control and Optimization, vol. 38, no. 3, pp. 751-766, 2000.

[44] Y. Shen and Y. Huang, "Global finite-time stabilisation for a class of nonlinear systems," International Journal of Systems Science, vol. 43, no. 1, pp. 73-78, 2012.

[45] G. H. Hardy, J. E. Littlewood, and G. Pólya, Inequalities, Cambridge University Press, Cambridge, UK, 1952.

[46] J. A. Moreno and M. Osorio, "A Lyapunov approach to secondorder sliding mode controllers and observers," in Proceedings of the 47th IEEE Conference on Decision and Control (CDC '08), pp. 2856-2861, December 2008.

[47] K. P. Bollino, High-Fidelity Real-Time Trajectory Optimizaiton for Reusable Launch Vehicles, Naval Postgraduate School, 2006.

[48] D. B. Doman, B. J. Gamble, and A. D. Ngo, "Quantized control allocation of reaction control jets and aerodynamic control surfaces," Journal of Guidance, Control, and Dynamics, vol. 32, no. 1, pp. 13-24, 2009. 


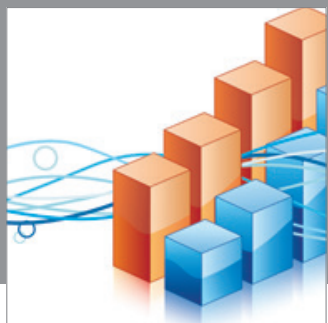

Advances in

Operations Research

mansans

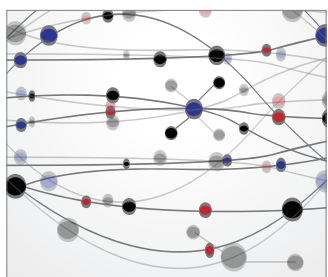

The Scientific World Journal
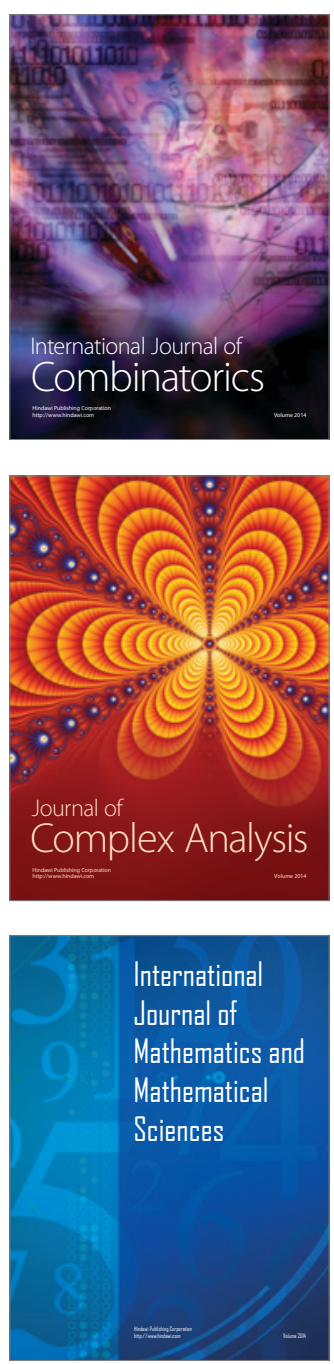
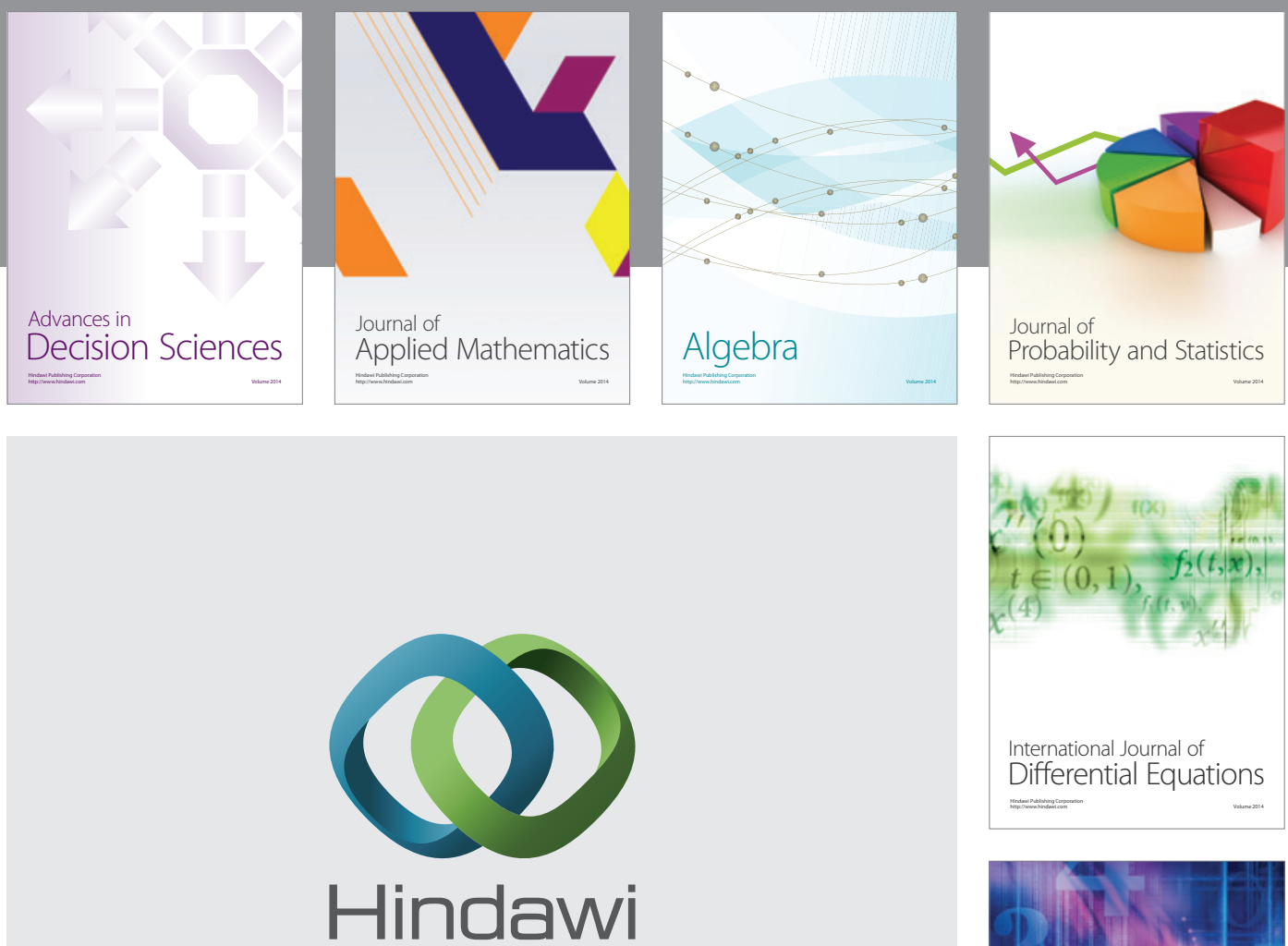

Submit your manuscripts at http://www.hindawi.com
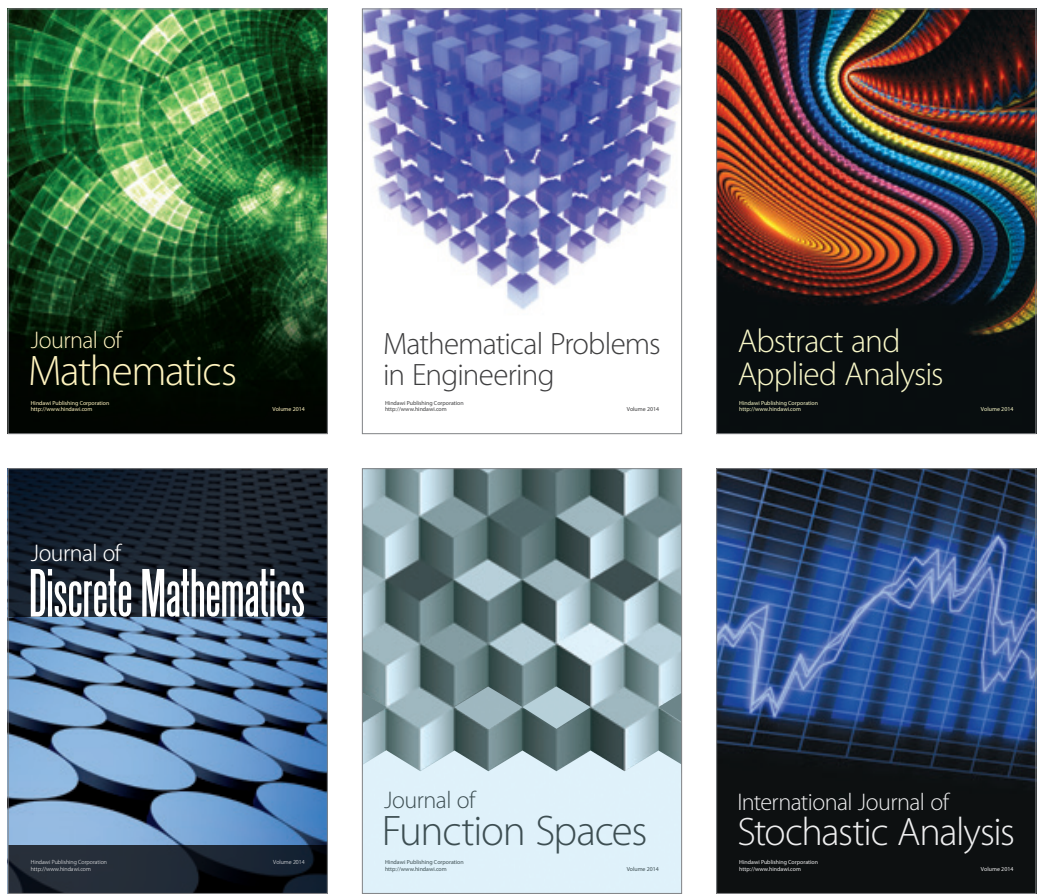

Journal of

Function Spaces

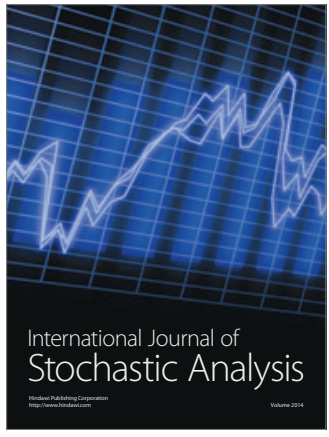

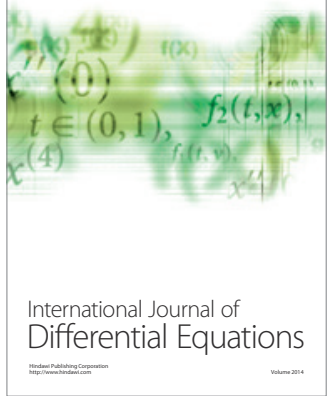
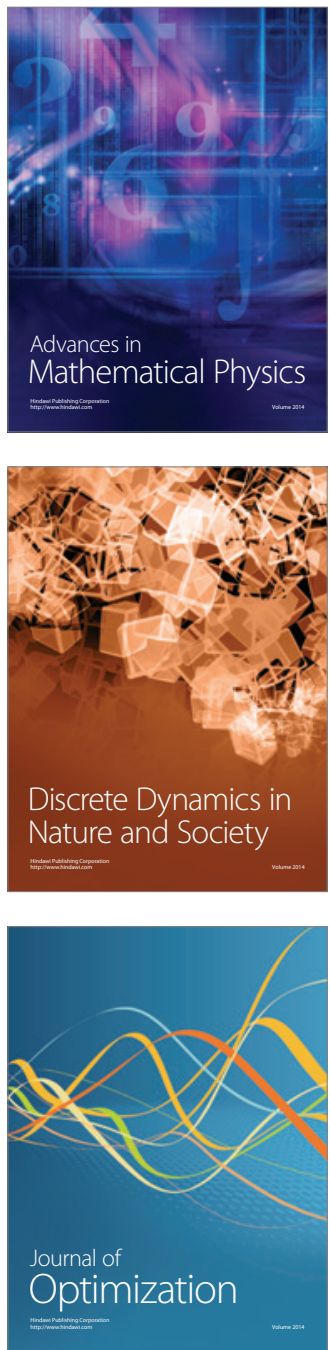